\title{
Impact of the Extent of Lymph Node Dissection on Precise Staging and Survivalin Clinical I-II Pure-Solid Lung Cancer Undergoing Lobectomy
}

Donglai Chen, MD ${ }^{1, *}$; Yiming Mao, MD, PhD ${ }^{2, *}$; Junmiao Wen, MDD,4,*; Jian Shu, MD5,6; Fei Ye, MD ${ }^{5,7}$; Yunlang She, MD; Qifeng Ding, MD5; Li Shi, MD5; Tao Xue, MD, PhD'; Min Fan, MD, PhD ${ }^{3,4, t}$; Yongbing Chen, MD, $\mathrm{PhD}^{5, t}$; and Chang Chen, MD, $\mathrm{PhD}^{1,+}$

\begin{abstract}
Background: This study sought to determine the optimal number of examined lymph nodes (ELNs) and examined node stations (ENSs) in patients with radiologically pure-solid non-small cell lung cancer (NSCLC) who underwent lobectomy and ipsilateral lymphadenectomy by investigating the impact of ELNs and ENSs on accurate staging and long-term survival. Materials and Methods: Data from 6 institutions in China on resected clinical stage I-II (cl-II) NSCLCs presenting as pure-solid tumors were analyzed for the impact of ELNs and ENSs on nodal upstaging, stage migration, recurrence-free survival (RFS), and overall survival (OS). Correlations between different endpoints and ELNs or ENSs were fitted with a LOWESS smoother, and the structural break points were determined by Chow test. Results: Both ELNs and ENSs were identified as independent prognostic factors for OS (ENS hazard ratio [HR], 0.690; $95 \% \mathrm{Cl}$, 0.597-0.797; $P<.001 ; E L N ~ H R, 0.950 ; 95 \% \mathrm{Cl}, 0.917-0.983 ; P=.004)$ and RFS (ENS HR, 0.859; 95\% Cl, 0.793-0.931; $P<.001$; ELN HR, 0.960; 95\% Cl, $0.942-0.962 ; P<.001)$, which were also associated with postoperative nodal upstaging (ENS odds ratio [OR], 1.057; 95\% Cl, 1.002-1.187; $P=.004$; ELN OR, 1.186; 95\% Cl, 1.148-1.226; $P<.001)$. A greater number of ELNs and ENSs correlated with a higher accuracy of nodal staging and a lower probability of stage migration. Cut-point analysis revealed an optimal cutoff of $18 \mathrm{LNs}$ and 6 node stations for stage cl-Il pure-solid NSCLCs, which were validated in our multi-institutional cohort. Conclusions: Extensive examination of $\mathrm{LNs}$ and node stations seemed crucial to predicting accurate staging and survival outcomes. A threshold of $18 \mathrm{LNs}$ and 6 node stations might be considered for evaluating the quality of LN examination in patients with stage cl-Il radiologically pure-solid NSCLCs.
\end{abstract}

J Natl Compr Canc Netw 2021;19(4):393-401 doi: 10.6004/jnccn.2020.7635

\footnotetext{
${ }^{1}$ Department of Thoracic Surgery, Shanghai Pulmonary Hospital, Tongji University School of Medicine, Shanghai; ${ }^{2}$ Department of Thoracic Surgery, Suzhou Kowloon Hospital Shanghai Jiaotong University School of Medicine, Suzhou; ${ }^{3}$ Department of Radiation Oncology, Fudan University Shanghai Cancer Center, Shanghai; ${ }^{4}$ Department of Oncology, Shanghai Medical College, Fudan University, Shanghai; ${ }^{5}$ Department of Thoracic Surgery, the Second Affiliated Hospital of Soochow University, Suzhou; ${ }^{6}$ Department of Thoracic Surgery, Taicang Affiliated Hospital of Soochow University, the First People's Hospital of Taicang, Taicang; ${ }^{7}$ Department of Thoracic Surgery, Hai'an Hospital Affiliated to Nantong University, Hai'an; and ${ }^{8}$ Department of

Cardiothoracic Surgery, Zhongda Hospital Southeast University, Nanjing, China.

*These authors contributed equally to this study.

tThese authors contributed equally as co-senior authors.
}

\section{Background}

With the introduction of CT screening for lung cancer and the extensive use of low-dose CT, the detection rate of early-stage non-small cell lung cancer (NSCLC) has increased remarkably. ${ }^{1,2}$ Early-stage NSCLCs can manifest as either subsolid or solid tumors on CT scans. Notably, lymph node (LN) involvement is much more frequently observed in radiologically solid tumors than in subsolid tumors. ${ }^{3-6}$ It has previously been reported that lymphadenectomy is unnecessary for lung cancer presenting as pure ground-glass nodules, ${ }^{6}$ which underscores the urgent need for a proposal for LN management of radiologically pure-solid NSCLCs.

In recent years, increasingly more researchers have been interested in the determination of an optimal number of examined LNs (ELNs). ${ }^{7-9}$ Liang et $\mathrm{al}^{7}$ recommended 16 ELNs as the cut point for evaluating the quality of LN examination or prognostic stratification postoperatively in patients with declared node-negative disease by analyzing the SEER database and a Chinese multi-institutional registry. Another study using the National Cancer Database found that 8 to 11 nodes should be examined in patients with stage I NSCLC for accurate staging and favorable outcomes. ${ }^{8}$ Similar results were observed in other studies in which removal of at least 10 nodes was found to be associated with better survival. ${ }^{10,11}$ Regrettably, neither the radiologic features of the primary tumors nor the examined node stations (ENSs) could be detailed in these studies.

For early-stage resectable NSCLCs, N1 and N2 node resection and mapping should be a routine component of lung cancer resections, with a minimum of $3 \mathrm{~N} 2$ nodes sampled or a complete LN dissection. ${ }^{12}$ Currently, ipsilateral systematic lymphadenectomy (SLND) in hilar and mediastinal stations, with 3 groups of $\mathrm{N} 1$ and $\mathrm{N} 2$

See JNCCN.org for supplemental online content. 
nodes examined, respectively, remains the overall standard. ${ }^{13,14}$ In addition, the LN map proposed by the International Association for the Study of Lung Cancer (IASLC) recommends that $\geq 1$ nodes should be sampled from all mediastinal stations, which for right-sided tumor-bearing lobes are $2 \mathrm{R}, 4 \mathrm{R}, 7,8$, and 9 and for the left side are 4L, 5, 6, 7, 8, and 9. ${ }^{15}$ However, another point of view based on several studies suggested that lobe-specific lymph node dissection (LSD) is equivalent to SLND in early-stage NSCLC. ${ }^{14,16,17}$ Therefore, it is important to determine the threshold of ELNs and ENSs for early-stage NSCLC, especially for radiologically pure-solid NSCLC.

To address these unresolved issues, we performed analyses of data collected from 6 institutions in China. By including information on both ELNs and ENSs, we assessed the relationships between the extent of LN dissection and long-term survival and pathologic upstaging. Advanced statistical methods were applied to determine optimal thresholds for ELNs and ENSs in patients with pure-solid clinical stage I-II (cI-II) NSCLC.

\section{Materials and Methods}

\section{Patient Selection}

This study evaluated patients with pure-solid cTla-2 bN0-1M0 NSCLCs who underwent R0 pulmonary resection at 6 medical centers in China between January 2010 and October 2015 (Shanghai Pulmonary Hospital, Tongji University School of Medicine, Suzhou Kowloon Hospital Shanghai Jiaotong University School of Medicine, Second Affiliated Hospital of Soochow University, Taicang Affiliated Hospital of Soochow University, Hai' an Hospital Affiliated to Nantong University, and Zhongda Hospital Southeast University). PET/CT was performed for clinical staging as necessary during the study period. Ethical approval was obtained from the participating institutions through their respective Institutional Review Boards. In cases in which individual patient consent was not identified, the chairperson of the ethics committee waived the need for patient consent.

In our study, a radiologic pure-solid tumor was defined as a lung tumor that only showed consolidation without a ground-glass opacity (GGO) component on thin-section CT. ${ }^{18}$ The definitions of solid component and GGO component were in line with those in a previous study. ${ }^{18}$ Tumor size was measured as the largest axial diameter of an area having increased opacification that completely obscured bronchial and vascular structures on the lung window setting (level, -500 Hounsfield units [HU]; width, 1,350 HU). Clinical T, N, and M stages were diagnosed according to the 8th edition of the TNM staging system for lung cancer. ${ }^{19}$ Clinical N1 stage was defined as having LNs with a short-axis diameter $>1 \mathrm{~cm}$ on CT scan or FDG uptake greater than that of surrounding normal structures on PET in stations 10, 11, 12, and $13 .{ }^{14}$ There were 4 main exclusion criteria: (1) multiple NSCLCs, (2) mediastinal LNs with a short-axis diameter $>1 \mathrm{~cm}$ on a CT scan or FDG uptake greater than that of surrounding normal structures on PET, (3) lesions pathologically diagnosed as adenocarcinoma in situ, minimally invasive adenocarcinoma, or benign disease, or (4) need for pneumonectomy, sleeve resection, sublobar resection, or bilobectomy. For all included patients, findings of preoperative CT were reviewed by the authors (D.C., Y.M., and J.W.). If disagreement occurred, discussion was held until a consensus was reached. The postoperative follow-up lasted until April 2020. In total, 1,205 patients were included in the study.

\section{Information on Harvested LNs and Nodal Status}

LNs were dissected en bloc with adipose tissue as far as possible, and all harvested LNs were classified according to the IASLC nodal map. ${ }^{15}$ With respect to the handling of N1 LNs, surgeons selectively collected LNs from stations 10 to 12 during surgery and then handed over the lung specimen to the pathologists. Because there was no standardized protocol for intrapulmonary LN dissection during the study period, segmental and subsegmental stations 13 and 14, respectively, were retrieved at the pathologist's discretion. Both the number and status of harvested LNs and node stations, respectively, were collected from each patient. ELNs were defined as the total number of examined LNs in the specimens. ENSs were defined as the total number of examined node stations. A heat map approach was applied to exhibit the nodal metastasis pattern according to the tumor locations, which reflected the cumulative number of patients with positive LNs at each node station.

\section{Recurrence and Overall Survival as Endpoints}

All patients were observed from the date of surgery after resection. In the first 2 years, follow-up procedures included chest radiographs; blood tests, including measurements of tumor markers every 3 months; and chest CT with or without contrast every 6 months. Subsequently, chest radiographs were performed every 6 months, and chest CT with or without contrast was performed every year. Further examinations were performed, including with brain MRI and bone scintigraphy, when any sign or symptom of tumor recurrence was detected. Locoregional recurrence was defined as tumor recurrence in the ipsilateral hemithorax, including the resection margin; ipsilateral lung; or hilum and mediastinal LNs. Distant metastasis was defined as tumor recurrence in the pleura, contralateral hemithorax, 


\begin{tabular}{|c|c|c|c|c|c|c|}
\hline Characteristic & Sig & HR $(95 \% \mathrm{Cl})$ & $P$ Value $^{a}$ & Sig & $\mathrm{HR}(95 \% \mathrm{Cl})$ & $P$ Value $^{\mathrm{a}}$ \\
\hline ENS (as a continuous variable) & $<.001$ & $0.690(0.597-0.797)$ & & $<.001$ & $0.859(0.793-0.931)$ & \\
\hline ELN (as a continuous variable) & .004 & $0.950(0.917-0.983)$ & & $<.001$ & $0.960(0.942-0.962)$ & \\
\hline LNR & .038 & $2.174(1.008-9.643)$ & & .036 & $2.688(1.005-6.571)$ & \\
\hline Operated side (left vs right) & .088 & $1.367(0.955-1.956)$ & & .694 & $1.050(0.822-1.341)$ & \\
\hline Tumor location (lower and middle vs upper) & & & .215 & & & .153 \\
\hline Middle lobe vs upper lobe & .774 & $1.110(0.543-2.270)$ & & .061 & $0.650(0.414-1.021)$ & \\
\hline Lower lobe vs upper lobe & .093 & $0.701(0.463-1.061)$ & & .442 & $0.914(0.726-1.150)$ & \\
\hline $\mathrm{pN}$ & & & $<.001$ & & & $<.001$ \\
\hline N1 vs N0 & $<.001$ & $5.265(3.201-8.659)$ & & $<.001$ & $4.141(3.110-5.513)$ & \\
\hline N2 vs NO & $<.001$ & $6.224(3.792-10.217)$ & & $<.001$ & $7.865(6.032-10.255)$ & \\
\hline pM (M1 vs M0) & $<.001$ & $53.357(11.877-239.709)$ & & $<.001$ & $33.192(8.027-137.242)$ & \\
\hline Histology (others, LCC, ASC, ADC vs SCC) & & & .376 & & & .034 \\
\hline$A D C$ vs $S C C$ & .850 & $1.050(0.633-1.743)$ & & .003 & $1.669(1.184-2.353)$ & \\
\hline ASC vs SCC & .178 & $1.903(0.746-4.8520$ & & .032 & $2.147(1.069-4.318)$ & \\
\hline LCC vs SCC & .147 & $1.989(0.785-5.043)$ & & .112 & $1.871(0.864-4.053)$ & \\
\hline Others vs SCC & .642 & $1.420(0.323-6.240)$ & & .734 & $1.191(0.434-3.275)$ & \\
\hline Adjuvant chemotherapy (yes vs no) & .574 & $1.143(0.716-1.825)$ & & & & \\
\hline Adjuvant radiotherapy (yes vs no) & .104 & $1.569(0.912-2.700)$ & & .969 & $0.993(0.700-1.409)$ & \\
\hline
\end{tabular}

Abbreviations: ADC, adenocarcinoma; ASC, adenosquamous carcinoma; ELN, examined lymph node number; ENS, examined node station; HR, hazard ratio; LASSO, least absolute shrinkage and selection operator; LCC, large cell carcinoma; LNR, lymph node ratio (ratio of metastatic lymph nodes to total number of lymph nodes examined); $p$, pathologic; SCC, squamous cell carcinoma; Sig, significance; VATS, video-assisted thoracoscopic surgery.

alnteraction test $P$ value.

or extrathoracic organs. Recurrence-free survival (RFS) was defined as the time from surgery until local or distant recurrence. Overall survival (OS) was defined as the time from surgery until all-cause death.

\section{Statistical Analysis}

\section{Multivariable Regression Analyses}

Theoretically, ELNs and ENSs are highly correlated with the number of positive LNs and the status of node stations, which in turn are highly correlated with $\mathrm{N}$ stage. To address the redundancy and multicollinearity among the variables in an overfitting model, we first performed a least absolute shrinkage and selection operator (LASSO) regression analysis ${ }^{20}$ to screen and shrink the data described as in our previous studies, ${ }^{21,22}$ which could achieve variant reduction and selection through a tuning parameter $(\lambda)$. Pearson's chi-square test was used to compare categorical variables, and an independent sample $t$ test was used to compare the continuous variables between different groups.

The log-rank test and the Cox proportional hazards regression model were used to determine the effect of ELNs and ENSs on survival, which were adjusted for 
other significant prognostic factors. ${ }^{7}$ To verify our assumption that more ELNs and ENSs present a greater opportunity to identify positive LNs, we performed a logistic regression analysis to detect the predictors associated with postoperative nodal upstaging. In addition, stage migration was assessed by correlating the ELNs and ENSs and the proportion of each nodal stage category (node-negative vs node-positive) by using a binary logistic regression model after adjusting for other potential confounders associated with examined nodes or nodal stage before or during surgery. ${ }^{7}$

\section{Accuracy of Number of Involved LNs and Node Stations} To evaluate the accuracy of involved LNs and node stations, we created mathematical models of the numbers of nodes and stations examined, respectively, by using hypergeometric distribution and the Bayes theorem according to previous studies. ${ }^{7,23}$ In addition, sensitivity analyses were conducted to evaluate whether the association between ELNs and OS and RFS was affected by outliers (probably caused by fragmented LNs). ${ }^{8}$

\section{Fitting of Curves and Determination of Structural Break Points}

The curves of odds ratios (ORs; stage migration) and hazard ratios (HRs; OS) of each ELN and ENS compared with one ELN or ENS (as a reference), in addition to the curves of mean positive number and probability of undetected positive LNs, were fitted by using a LOWESS (locally weighted scatterplot smoothing) smoother with a bandwidth of $2 / 3$ (default). ${ }^{7}$ Structural break points were then determined by Chow test, and the break points were considered the threshold of clinical impact. ${ }^{7}$ In addition, to assess whether the number of LNs needed to optimize survival was consistent with the number needed to optimize accurate nodal staging, we plotted the frequency of patients with at least one positive LN for each LN count using locally weighted least-squares smoothing. ${ }^{24}$

All clinical data are shown as either mean \pm SD or number (percent). A 2-sided $P$ value $<.05$ was considered statistically significant. Statistical analyses were performed using R version 3.5.3 (R Foundation for Statistical Computing) and SPSS Statistics, version 25.0 (IBM Corp). The heat map and survival curves were drawn with Prism 7.0 software (GraphPad Software).

\section{Results}

Patient Characteristics and Distribution of

\section{ELNs and ENSs}

Overall, 1,205 patients with cTla-2bN0-1M0 NSCLCs manifesting as radiologically pure-solid tumors who underwent lobectomy and ipsilateral lymphadenectomy at 6 medical centers were recruited. Median follow-up

\begin{tabular}{|c|c|c|c|}
\hline Characteristic & Sig & OR $(95 \% \mathrm{Cl})$ & $\begin{array}{l}P \\
\text { Value }^{a}\end{array}$ \\
\hline ENS (as a continuous variable) & .004 & $1.057(1.002-1.187)$ & \\
\hline ELN (as a continuous variable) & $<.001$ & $1.186(1.148-1.226)$ & \\
\hline Sex (male vs female) & .959 & $0.991(0.688-1.425)$ & \\
\hline Smoking history (yes vs no) & .345 & $0.834(0.573-1.215)$ & \\
\hline Operated side (left vs right) & .001 & $1.660(1.229-2.242)$ & \\
\hline Tumor location & & & .001 \\
\hline Middle lobe vs upper lobe & .003 & $2.614(1.400-4.881)$ & \\
\hline Lower lobe vs upper lobe & .060 & $0.733(0.530-1.014)$ & \\
\hline pT & & & .003 \\
\hline T2 vs T1 & $<.001$ & $1.725(1.274-2.334)$ & \\
\hline T3 vs T1 & .528 & $1.597(0.373-6.837)$ & \\
\hline T4 vs T1 & .125 & $5.280(0.631-44.162)$ & \\
\hline Histology & & & .001 \\
\hline$A D C$ vs $S C C$ & .001 & $2.101(1.366-3.229)$ & \\
\hline ASC vs SCC & .383 & $0.603(0.194-1.879)$ & \\
\hline LCC vs SCC & .012 & $3.822(1.338-10.916)$ & \\
\hline Others vs SCC & .351 & $1.846(0.509-6.688)$ & \\
\hline Grade of differentiation & & & .002 \\
\hline Moderate vs well & .043 & $2.287(1.028-5.091)$ & \\
\hline Poor vs well & .001 & $4.188(1.797-9.759)$ & \\
\hline Others vs well & .051 & $2.534(0.997-6.439)$ & \\
\hline $\begin{array}{l}\text { Surgical approach (thoracotomy } \\
\text { vs VATS) }\end{array}$ & .006 & $1.825(1.189-2.801)$ & \\
\hline
\end{tabular}

Abbreviations: ADC, adenocarcinoma; ASC, adenosquamous carcinoma; ELN examined lymph node number; ENS, examined node stations; LCC, large cell carcinoma; LL, lower limit of $95 \% \mathrm{Cl}$; OR, odds ratio; p, pathologic; SCC, squamous cell carcinoma; Sig, significance; UL, upper limit of $95 \% \mathrm{Cl}$; VATS, video-assisted thoracoscopic surgery.

alnteraction test $P$ value.

time was 68 months. Baseline characteristics of the patient cohort are summarized in supplemental eTable 1 (available with this article at JNCCN.org).

The distribution of ELNs and ENSs in our cohort is shown in supplemental eFigure 1 . We used a heat map to assess whether nodal metastasis was lobe-specific in pure-solid NSCLCs (supplemental eFigure 2). Our results revealed that tumor location was not a predictor of involved zones, which highlighted the importance of extensive examination of LNs at different positions in the resected specimens.

\section{Identification of ELNs and ENSs as Prognostic Factors}

Based on numerous clinicopathologic variables with mutual collinearity, both ELNs and ENSs were initially identified as potential survival-related factors by LASSO regression analysis (Table 1). Variants included in the LASSO regression model are listed in supplemental 
eTable 2. Multivariate Cox analysis was performed to further confirm ENSs and ELNs as prognostic factors for both OS (ENS HR, 0.690; 95\% CI, 0.597-0.797; $P<.001$; ELN HR, 0.950; 95\% CI, 0.917-0.983; $P=.004)$ and RFS (ENS HR, 0.859; 95\% CI, 0.793-0.931; $P<.001$; ELN HR, 0.960; 95\% CI, 0.942-0.962; $P<.001$ ), respectively. To eliminate potential bias from the count of LN fragments, a sensitivity analysis was performed by limiting ELNs to $<20$, and ELN number remained statistically significant for both OS (HR, 0.935; 95\% CI, 0.897-0.974; $P=.002$ ) and RFS (HR, 0.941; 95\% CI, 0.925-0.956; $P<.001$ ).

\section{Association of ELNs and ENSs With Nodal Upstaging and Stage Migration}

Mean ELNs and ENSs differed significantly within subgroups of T staging, $\mathrm{N}$ staging, and tumor location in our cohort (supplemental eFigure 3). Because a number of confounding factors are associated with occult mediastinal LN metastasis, we established a multivariate logistic regression model after performing a LASSO regression analysis. Variants included in the LASSO regression

A

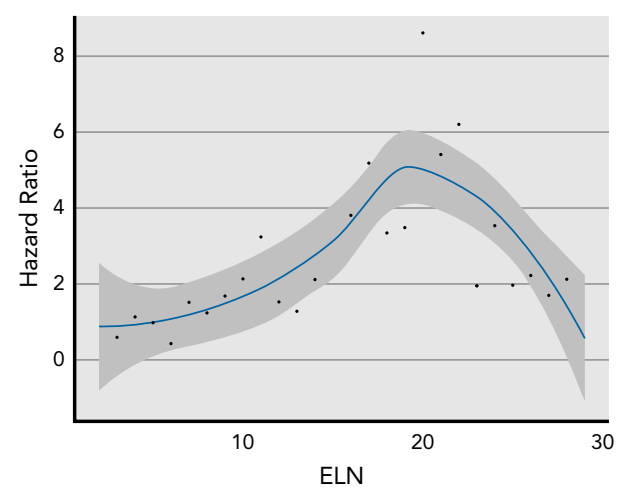

C

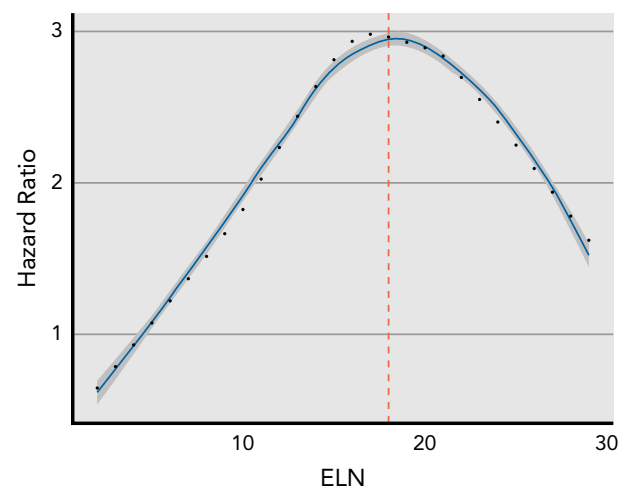

model are listed in supplemental eTable 3 . As shown in Table 2, both ELNs and ENSs were found to be independent predictors of postoperative nodal upstaging (ENS OR, 1.057; 95\% CI, 1.002-1.187; $P=.004$; ELN OR, 1.186; 95\% CI, 1.148-1.226; $P<.001$ ).

In addition, the patient cohort was also used to estimate the empirical distributions of the number of positive LNs; these results were then used to calculate the probabilities of having more positive nodes than observed (supplemental eTables 4-7). As expected, a greater number of harvested LNs and node stations correlated with a higher accuracy of nodal staging (supplemental eTables 4 and 5) and a lower probability of stage migration (supplemental eTables 6 and 7).

\section{Cut Point Analysis for Optimal ELNs and ENSs}

Figures 1 and 2 exhibit the fitting curves and corresponding structural break points for HRs of OS and RFS in radiologically pure-solid NSCLCs. Our data reveal that the cut points of ELNs and ENSs for OS and RFS are almost in agreement.
B

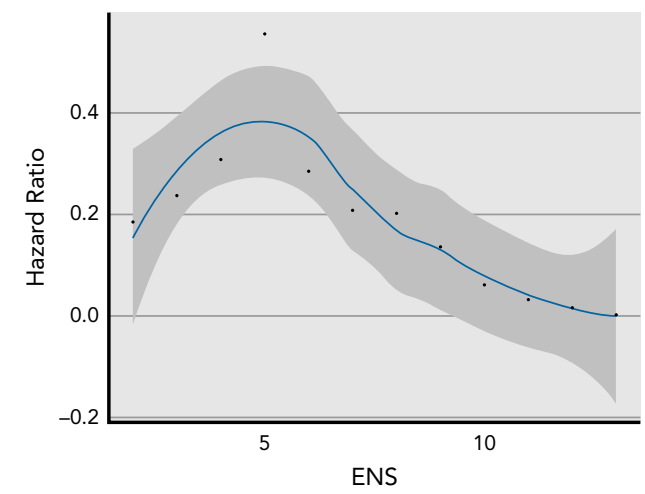

D

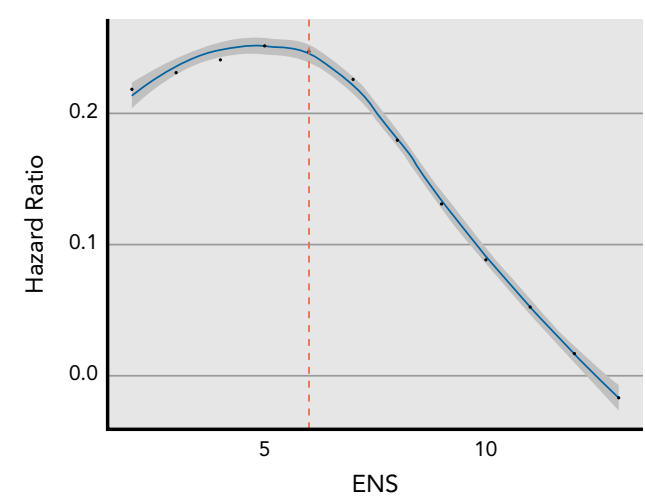

Figure 1. LOWESS smoother-fitting curves of $(A, B) O S$ and $(C, D)$ determination of structural break points using the Chow test. The fitting bandwidth was $2 / 3$. OS was estimated by using the Cox proportional hazards regression model after LASSO regression analysis.

Abbreviations: ELN, examined lymph node; ENS, examined node station; LASSO, least absolute shrinkage and selection operator; LOWESS, locally weighted scatterplot smoothing; OS, overall survival. 
A

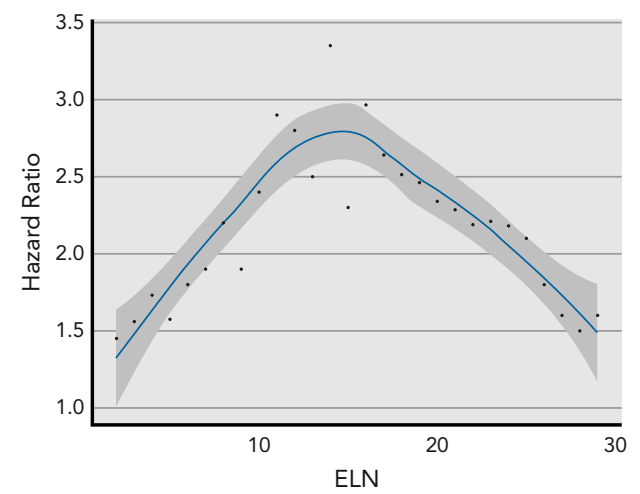

C

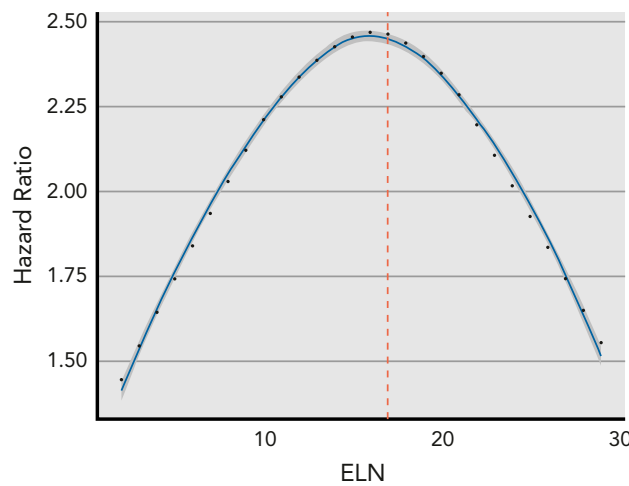

B

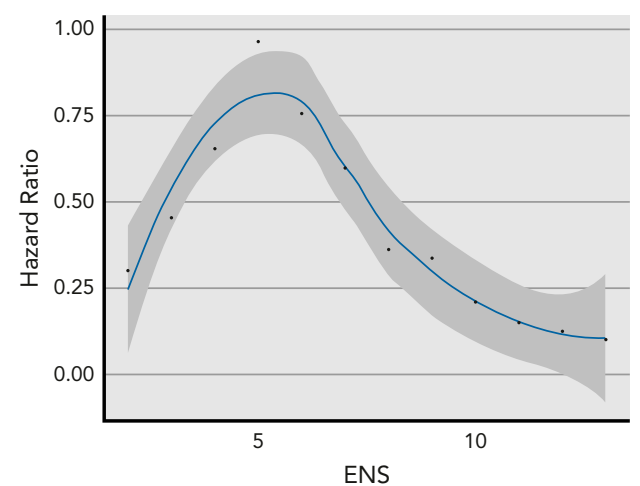

D

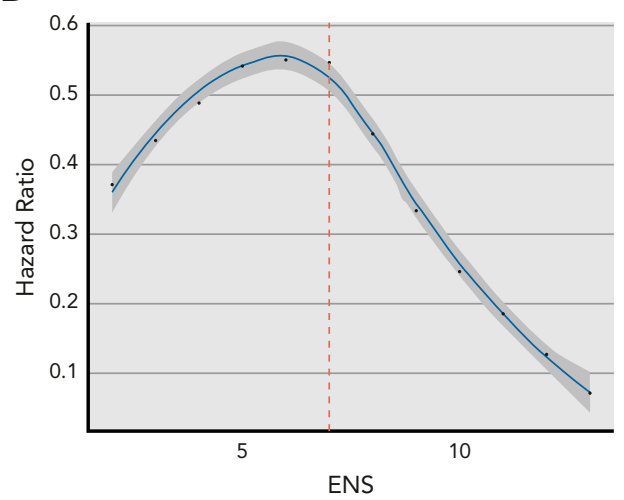

Figure 2. LOWESS smoother-fitting curves of (A, B) RFS and (C, D) determination of structural break points using the Chow test. The fitting bandwidth was $2 / 3$. RFS was estimated by using the Cox proportional hazards regression model after LASSO regression analysis.

Abbreviations: ELN, examined lymph node; ENS, examined node station; LASSO, least absolute shrinkage and selection operator; LOWESS, locally weighted scatterplot smoothing; RFS, recurrence-free survival.

To determine the cut points of ELNs and ENSs for stage migration, we plotted the fitting curves and corresponding structural break points for the OR of stage migration (Figure 3). As shown in Figure 3C, D, the probability of stage migration reaches a cut point at 18 ELNs and 7 ENSs. We also plotted the probability of finding at least one positive LN by the ELNs and ENSs, respectively, using locally weighted least squares smoothing (supplemental eFigure 4). The probability of finding a positive LN reaches a cut point at 6 ENSs and 15 harvested LNs, respectively (supplemental eFigure 4). The structural break points of the estimated probabilities of having positive nodes or stations in patients with node-negative disease were also determined (supplemental eFigure 5).

Because OS is the most important issue, we selected the structural break point of OS as the cut point. Therefore, we used cutoff values of 18 LNs and 6 stations as the optimal ELNs and ENSs for patients with radiologically pure-solid NSCLCs.

The cut point was then validated in our multicenter cohort. Survival analysis revealed that all-cause mortality of patients was significantly reduced with at least $18 \mathrm{LNs}$ or 6 node stations (supplemental eFigure $6 \mathrm{~A}, \mathrm{~B}$ ). Similar results were also observed in patients with declared node-negative disease (supplemental eFigure 6C, D).

\section{Discussion}

The heterogeneity in examined stations and the number of LNs counted at each station could be due to a series of factors, including surgeon skills and preferences, ${ }^{13}$ individual variations among patients' LN maps, locations of LNs, radiologic features of the lesions, and performance of en bloc resection. For NSCLC, the current NCCN Clinical Practice Guidelines in Oncology (NCCN Guidelines) ${ }^{12}$ have not provided a recommended minimum number of harvested nodes, which might be because of the fragile structure of the LN capsule and the surrounding sheath. ${ }^{13}$ Meanwhile, the NCCN Guidelines recommend that patients should have a minimum of 3 $\mathrm{N} 2$ stations sampled, ${ }^{12}$ although no direct supporting evidence was available. Notably, LSD has increasingly been performed in recent years, in which the following 
A

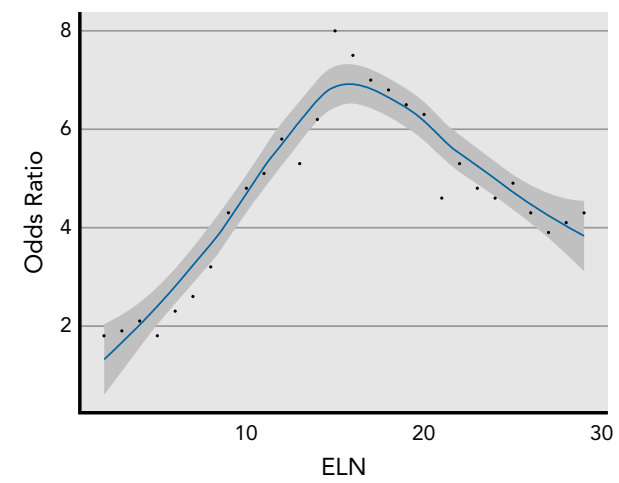

c

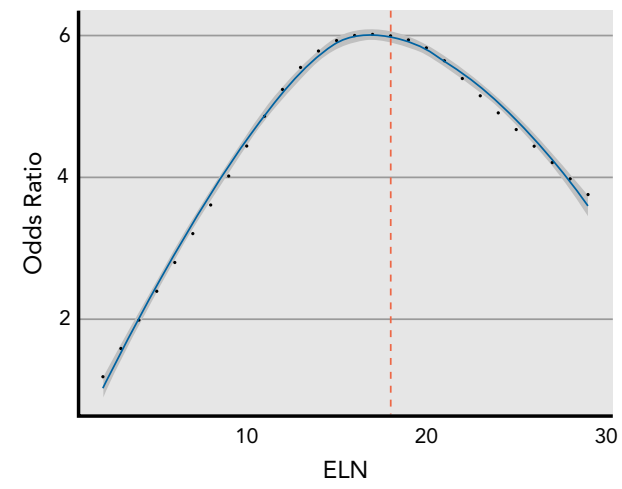

B

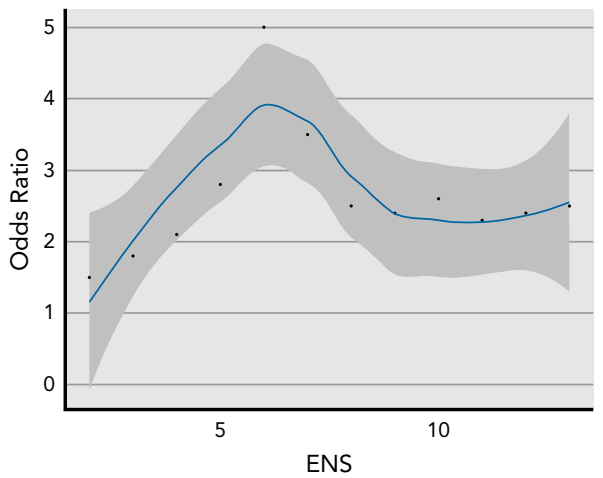

D

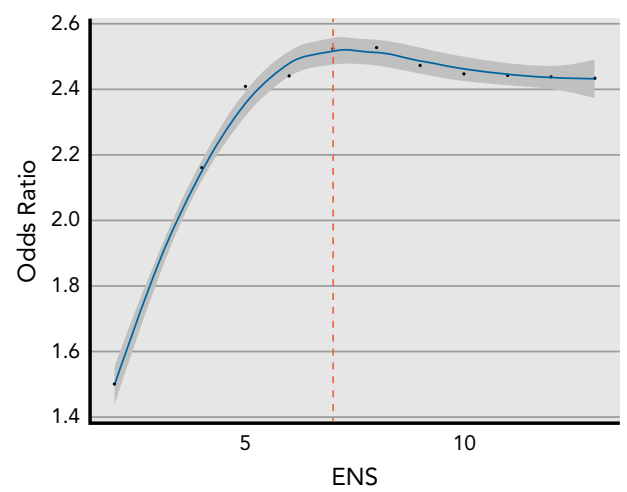

Figure 3. LOWESS smoother-fitting curves of $(A, B)$ stage migration and $(C, D)$ determination of structural break points using the Chow test. The fitting bandwidth was $2 / 3$. Stage migration was estimated by using the Cox proportional hazards regression model after LASSO regression analysis.

Abbreviations: ELN, examined lymph node; ENS, examined node station; LASSO, least absolute shrinkage and selection operator; LOWESS, locally weighted scatterplot smoothing.

stations were dissected routinely: $2 \mathrm{R}$ and $4 \mathrm{R}$ for right upper-lobe tumors; 4L, 5, and 6 for left upper-lobe tumors; and 7, 8, and 9 for lower-lobe tumors on both sides. ${ }^{25}$ Moreover, the noninferiority of LSD compared with SLND was confirmed by a randomized phase III trial (JCOG1413) in 2018. ${ }^{25}$ The extensive use of LSD ${ }^{14,16,17,25}$ may support the minimum of $3 \mathrm{~N} 2$ stations examined in early-stage NSCLCs. However, these studies ${ }^{14,16,17,25}$ detailed neither the proportion of the included patients with NSCLCs manifesting as GGOs nor the number of harvested LNs. Therefore, the optimal number of examined stations and of LNs for radiologically pure-solid NSCLCs that indicate a higher risk of LN metastasis and a larger invasive size remains undetermined. ${ }^{26}$

Our study included a number of clinicopathologic characteristics, especially $\mathrm{cN}, \mathrm{pN}$, and $\mathrm{LN}$ status at each station. Interestingly, our initial findings revealed that involved LNs could be detected beyond the lobespecific zone of the primary tumor location (supplemental eFigure 2), which was similar to the observed results in other studies. ${ }^{27-29}$ The nodal metastasis patterns shown by the heat map (supplemental eFigure 2) also highlighted the irreplaceable role of SLND for operable pure-solid NSCLCs. By using LASSO regression analysis and multivariate logistic regression analysis, we identified both ELNs and ENSs as independent prognostic factors for OS and RFS. In addition, the stage migration analyses suggested that a larger number of ELNs and ENSs was associated with a higher proportion of more-advanced N-stage cases in the entire population (supplemental eTables 4-7). As illustrated, a more extensive examination of LNs and stations can reduce the risk of undetected positive LNs and involved stations (supplemental eTables 4-7), which may result in a more thorough elimination of remnants and proper delivery of adjuvant therapy to improve long-term survival. ${ }^{7}$ Moreover, we identified an optimal cutoff of $18 \mathrm{LNs}$ and 6 node stations for cTla-2bN0-1M0 pure-solid NSCLC (Figures 1-3). Interestingly, the optimal number of harvested LNs according to Liang et $\mathrm{al}^{7}$ was similar to that of our study. Another study concerning complete hilar and 
mediastinal lymphadenectomy claimed that the mean (SD) total number of harvested LNs was 17.4 (7.3), ${ }^{30}$ which also supported the cut point we found. Therefore, the threshold of ELNs might be considered as the reference index for defining inadequate $\mathrm{LN}$ sampling.

Our study is the largest one on lymphadenectomy in pure-solid NSCLCs using multi-institutional, real-world datasets with robust statistics. We sought to emphasize 2 major points. First, both ELNs and ENSs are associated with clinical outcomes and accurate staging in patients with pure-solid NSCLC receiving lobectomy and ipsilateral lymphadenectomy; therefore, a more extensive lymphadenectomy should be performed in patients with pure-solid NSCLC in case of occult LN metastasis. Second, surgeons and pathologists should establish criteria for evaluating the completeness of intraoperative LN management for pure-solid NSCLC.

We acknowledge some limitations of our study. First, the retrospective nature of our multicenter study might lead to selection and performance bias. Second, because fragmentation of nodal tissues was inevitable during the removal of LNs, unavoidable overestimation of ELNs probably had an interference effect on our analysis, even though the sensitivity analysis was used to mitigate the bias. To be specific, some of the patients in our cohort had $\geq 20$ LNs examined, which is uncommon in a standard resection for cN0-1 NSCLC.

\section{Conclusions}

Both ELNs and ENSs are associated with accurate staging and survival outcomes in radiologically pure-solid NSCLC. A threshold of 18 LNs and 6 stations might be considered for evaluating the quality of LN examination in patients with radiologically pure-solid clinical stage I-II NSCLC.

\section{Acknowledgments}

We wish to thank Yiting Zhou, postgraduate student in the Department of Epidemiology, School of Public Health, Medical College of Soochow University, and Yueping Shen, professor and director of the Department of Epidemiology, School of Public Health, Medical College of Soochow University, for providing statistical support. We also wish to thank Hang Su, doctoral candidate in the Department of Thoracic Surgery, Shanghai Pulmonary Hospital, Tongji University School of Medicine, and Wentao Yang, deputy director of Department of Thoracic Surgery, the Second Affiliated Hospital of Soochow University, for providing administrative support.

Submitted February 20, 2020; accepted for publication August 6, 2020. Published online January 28, 2021

Author contributions: Study concept and design: D. Chen, Mao, Wen, Xue, Fan, Y. Chen, C. Chen. Data acquisition: Shu, Ye, She, Ding, Shi, Xue. Data analysis and interpretation: D. Chen, Mao, Wen. Manuscript preparation: D. Chen, Mao, Wen, Shu, Ye, She, Ding, Shi. Critical revision: Xue, Fan, Y. Chen, C. Chen. Supervision: Fan, Y. Chen, C. Chen.

Disclosures: The authors have disclosed that they have not received any financial consideration from any person or organization to support the preparation, analysis, results, or discussion of this article.

Funding: This work was supported by projects from the Shanghai Hospital Development Center (SHDC12015116); the National Natural Science Foundation of China (81802256); the Science and Technology Commission of Shanghai Municipality (15411968400 and 14411962600); the Suzhou Key Laboratory of Thoracic Oncology (SZS201907); the Suzhou Key Discipline for Medicine (SZXK201803); the Municipal Program of People's Livelihood Science and Technology in Suzhou (SS2019061); Jiangsu Key Research and Development Plan (Social Development) Project (BE2020653); and Clinical Research Plan of Shanghai Hospital Development Center (SHDC2020CR3025B).

Correspondence: Chang Chen, MD, PhD, Department of Thoracic Surgery, Shanghai Pulmonary Hospital, Tongji University School of Medicine, 507 Zhengmin Road, Yangpu District, Shanghai 200433, China.

Email: chenthoracic@163.com;

Yongbing Chen, MD, PhD, Department of Thoracic Surgery, the Second Affiliated Hospital of Soochow University, 1055 Sanxiang Road, Suzhou 215004, China. Email: chentongt@sina.com; and

Min Fan, MD, PhD, Department of Radiation Oncology, Shanghai Cancer Center, Fudan University Shanghai Medical School, 270 Dong'an Road, and Department of Oncology, Shanghai Medical College, 131 Dong'an Road, Fudan University, Shanghai 200032. Email: minfan20141212@hotmail.com

\section{References}

1. Dai C, Shen J, Ren Y, et al. Choice of surgical procedure for patients with non-small-cell lung cancer $\leq 1 \mathrm{~cm}$ or $>1$ to $2 \mathrm{~cm}$ among lobectomy, segmentectomy, and wedge resection: a population-based study. J Clin Oncol 2016;34:3175-3182.

2. National Lung Screening Trial Research Team. Reduced lung-cancer mortality with low-dose computed tomographic screening. N Engl J Med 2011;365:395-409.

3. Wang $\mathrm{L}$, Jiang $\mathrm{W}$, Zhan $\mathrm{C}$, et al. Lymph node metastasis in clinical stage IA peripheral lung cancer. Lung Cancer 2015;90:41-46.

4. Hattori A, Suzuki K, Matsunaga T, et al. Is limited resection appropriate for radiologically "solid" tumors in small lung cancers? Ann Thorac Surg 2012;94:212-215.

5. Cho S, Song IH, Yang HC, et al. Predictive factors for node metastasis in patients with clinical stage I non-small cell lung cancer. Ann Thorac Surg 2013;96:239-245

6. Lin $\mathrm{YH}$, Chen $\mathrm{CK}$, Hsieh CC, et al. Lymphadenectomy is unnecessary for pure ground-glass opacity pulmonary nodules. J Clin Med 2020;9:672.

7. Liang $W, \mathrm{He} J$, Shen $Y$, et al. Impact of examined lymph node count on precise staging and long-term survival of resected non-small-cell lung cancer: a population study of the US SEER database and a Chinese multiinstitutional registry. J Clin Oncol 2017;35:1162-1170.
8. Dai J, Liu M, Yang Y, et al. Optimal lymph node examination and adjuvant chemotherapy for stage I lung cancer. J Thorac Oncol 2019;14:1277-1285.

9. David EA, Cooke DT, Chen Y, et al. Does lymph node count influence survival in surgically resected non-small cell lung cancer? Ann Thorac Surg 2017; 103:226-235.

10. Samayoa AX, Pezzi TA, Pezzi CM, et al. Rationale for a minimum number of lymph nodes removed with non-small cell lung cancer resection: correlating the number of nodes removed with survival in 98,970 patients. Ann Surg Oncol 2016;23(Suppl 5):1005-1011.

11. Bosch DE, Farjah F, Wood DE, et al. Regional lymph node sampling in lung carcinoma: a single institutional and national database comparison. Hum Pathol 2018;75:55-62.

12. Ettinger DS, Wood DE, Aisner DL, et al. NCCN Clinical Practice Guidelines in Oncology: Non-Small Cell Lung Cancer. Version 1.2021. Accessed December 1, 2020. To view the most recent version, visit NCCN.org

13. Zhong WZ, Liu SY, Wu YL. Numbers or stations: from systematic sampling to individualized lymph node dissection in non-small-cell lung cancer. J Clin Oncol 2017;35:1143-1145

14. Adachi H, Sakamaki K, Nishii T, et al. Lobe-specific lymph node dissection as a standard procedure in surgery for non-small cell lung cancer: a propensity score matching study. J Thorac Oncol 2017;12:85-93. 
15. Rusch WW, Asamura H, Watanabe H, et al. The IASLC Lung Cancer Staging Project: a proposal for a new international lymph node map in the forthcoming seventh edition of the TNM classification for lung cancer. J Thorac Oncol 2009;4:568-577.

16. Hishida T, Miyaoka E, Yokoi K, et al. Lobe-specific nodal dissection for clinical stage I and II NSCLC: Japanese multi-institutional retrospective study using a propensity score analysis. J Thorac Oncol 2016;11: 1529-1537.

17. Shapiro M, Kadakia S, Lim J, et al. Lobe-specific mediastinal nodal dissection is sufficient during lobectomy by video-assisted thoracic surgery or thoracotomy for early-stage lung cancer. Chest 2013;144: 1615-1621.

18. Hattori A, Suzuki K, Maeyashiki T, et al. The presence of air bronchogram is a novel predictor of negative nodal involvement in radiologically puresolid lung cancer. Eur J Cardiothorac Surg 2014;45:699-702.

19. Goldstraw P, Chansky K, Crowley J, et al. The IASLC Lung Cancer Staging Project: proposals for revision of the TNM stage groupings in the forthcoming (eighth) edition of the TNM classification for lung cancer. J Thorac Oncol 2016;11:39-51.

20. Li B, Cui Y, Diehn M, et al. Development and validation of an individualized immune prognostic signature in early-stage nonsquamous non-small cell lung cancer. JAMA Oncol 2017;3:1529-1537.

21. Chen $D$, Song $Y$, Zhang $F$, et al. Genome-wide analysis of lung adenocarcinoma identifies novel prognostic factors and a prognostic score. Front Genet 2019;10:493

22. Song $Y$, Chen $D$, Zhang $X$, et al. Integrating genetic mutations and expression profiles for survival prediction of lung adenocarcinoma. Thorac Cancer 2019;10:1220-1228.
23. Iyer RV, Hanlon A, Fowble B, et al. Accuracy of the extent of axillary nodal positivity related to primary tumor size, number of involved nodes, and number of nodes examined. Int J Radiat Oncol Biol Phys 2000;47: 1177-1183

24. Groth SS, Virnig BA, Whitson BA, et al. Determination of the minimum number of lymph nodes to examine to maximize survival in patients with esophageal carcinoma: data from the Surveillance Epidemiology and End Results database. J Thorac Cardiovasc Surg 2010;139:612-620.

25. Hishida T, Saji H, Watanabe SI, et al. A randomized phase III trial of lobespecific vs. systematic nodal dissection for clinical stage I-II non-small cell lung cancer (JCOG1413). Jpn J Clin Oncol 2018;48:190-194.

26. Mao R, She $Y, Z$ Zhu E, et al. A proposal for restaging of invasive lung adenocarcinoma manifesting as pure ground glass opacity. Ann Thorac Surg 2019;107:1523-1531.

27. Zheng $\mathrm{H}$, Wang LM, Bao F, et al. Re-appraisal of N2 disease by lymphatic drainage pattern for non-small-cell lung cancers: by terms of nodal stations, zones, chains, and a composite. Lung Cancer 2011;74: 497-503.

28. Riquet $\mathrm{M}$, Rivera $\mathrm{C}$, Pricopi $\mathrm{C}$, et al. Is the lymphatic drainage of lung cancer lobe-specific? A surgical appraisal. Eur J Cardiothorac Surg 2015; 47:543-549.

29. Liang RB, Yang J, Zeng TS, et al. Incidence and distribution of lobe-specific mediastinal lymph node metastasis in non-small cell lung cancer: data from 4511 resected cases. Ann Surg Oncol 2018;25 3300-3307.

30. Riquet $\mathrm{M}$, Legras $\mathrm{A}$, Mordant $\mathrm{P}$, et al. Number of mediastinal lymph nodes in non-small cell lung cancer: a Gaussian curve, not a prognostic factor. Ann Thorac Surg 2014;98:224-231. 
Supplemental online content for:

\section{Impact of the Extent of Lymph Node Dissection on Precise Staging and Survival in Clinical I-II Pure-Solid Lung Cancer Undergoing Lobectomy}

Donglai Chen, MD; Yiming Mao, MD, PhD; Junmiao Wen, MD; Jian Shu, MD; Fei Ye, MD; Yunlang She, MD; Qifeng Ding, MD; Li Shi, MD; Tao Xue, MD, PhD; Min Fan, MD, PhD; Yongbing Chen, MD, PhD; and Chang Chen, MD, PhD

J Natl Compr Canc Netw 2021;19(4):393-401

eFigure 1: Distribution of the Number of ELNs and ENSs in the Patient Cohort

eFigure 2: Distribution of Involved Node Stations According to Different Primary Tumor Locations in Radiologically Pure-Solid NSCLC

eFigure 3: Number of Examined Lymph Nodes and Node Stations Among Different T Stages, N Stages, and Tumor Locations

eFigure 4: Locally Weighted Least Squares Smoothing of Percentage of Patients With at Least One Positive Lymph Node or One Involved Node Station

eFigure 5: LOWESS Smoother Fitting Curves of Estimated Probabilities of Undetected Positive ELNs or ENSs and Determination of Structural Break Points With Use of the Chow Test in Patients With Declared Node-Negative Disease

eFigure 6: Stratification of OS at Cut Point of Number of ELNs and ENSs

eTable 1: Baseline Characteristics of the Patient Cohort

eTable 2: Variants in LASSO Regression Analysis of Screening Data in Survival Analysis

eTable 3: Variants in LASSO Regression Analysis of Screening Data in Multivariate Logistic Regression Analysis

eTable 4: Accuracy of Number of Positive Node Number

eTable 5: Accuracy of Number of Positive Station Number

eTable 6: Probability of Having M+1 or More Positive Nodes Given the Observation of M-Positive Nodes in $\mathrm{N}$-Resected Nodes

eTable 7: Probability of Having M+1 or More Positive Node Stations Given the Observation of M Stations With Positive Nodes in S-Examined Node Stations 
A

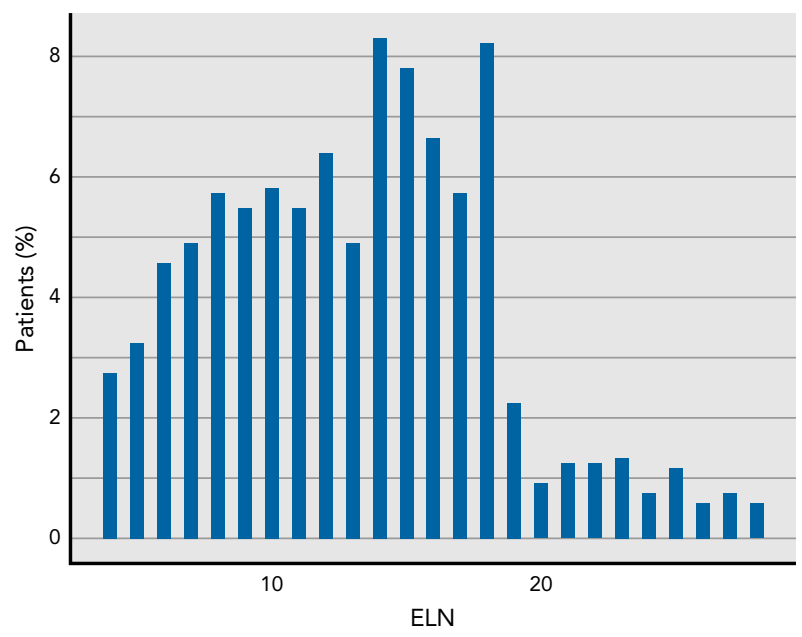

B

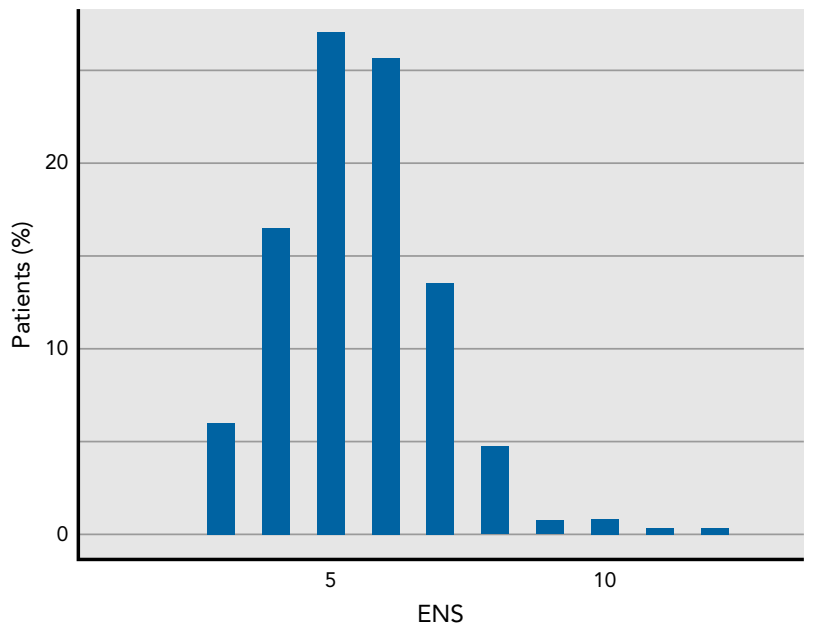

eFigure 1. Distribution of the number of (A) ELNs and (B) ENSs in the patient cohort. Abbreviations: ELN, examined lymph node; ENS, examined node station.
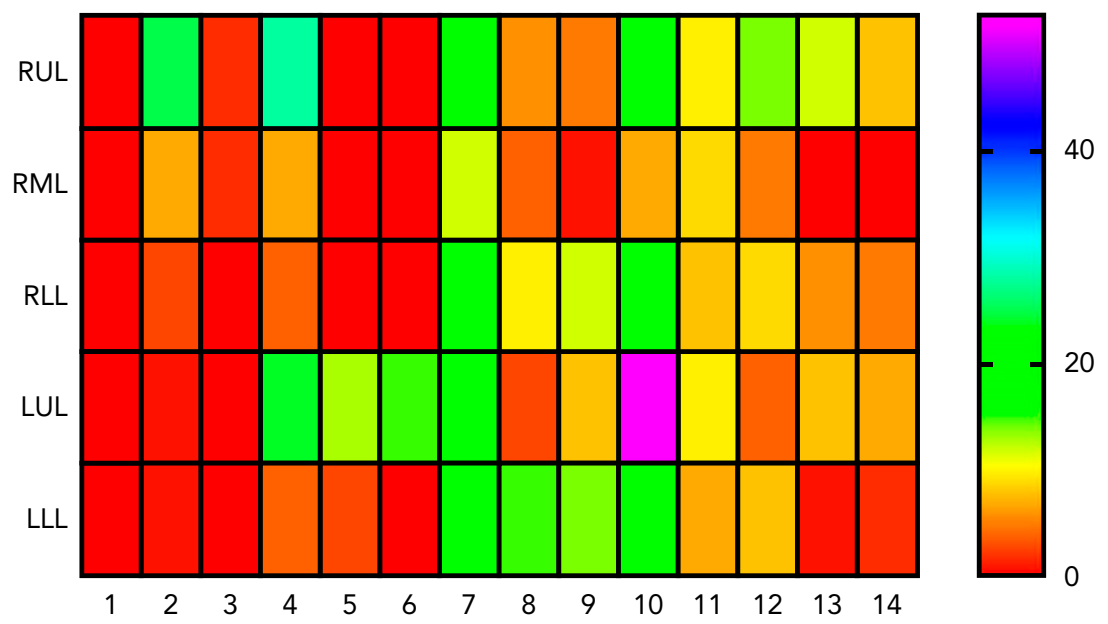

eFigure 2. Distribution of involved node stations according to different primary tumor locations in radiologically pure-solid NSCLC. Colors in the right bar correspond to numbers of patients with node metastasis.

Abbreviations: LLL, left lower lobe; LUL, left upper lobe; NSCLC, non-small cell lung cancer; RLL, right lower lobe; RML, right middle lobe; RUL, right upper lobe. 

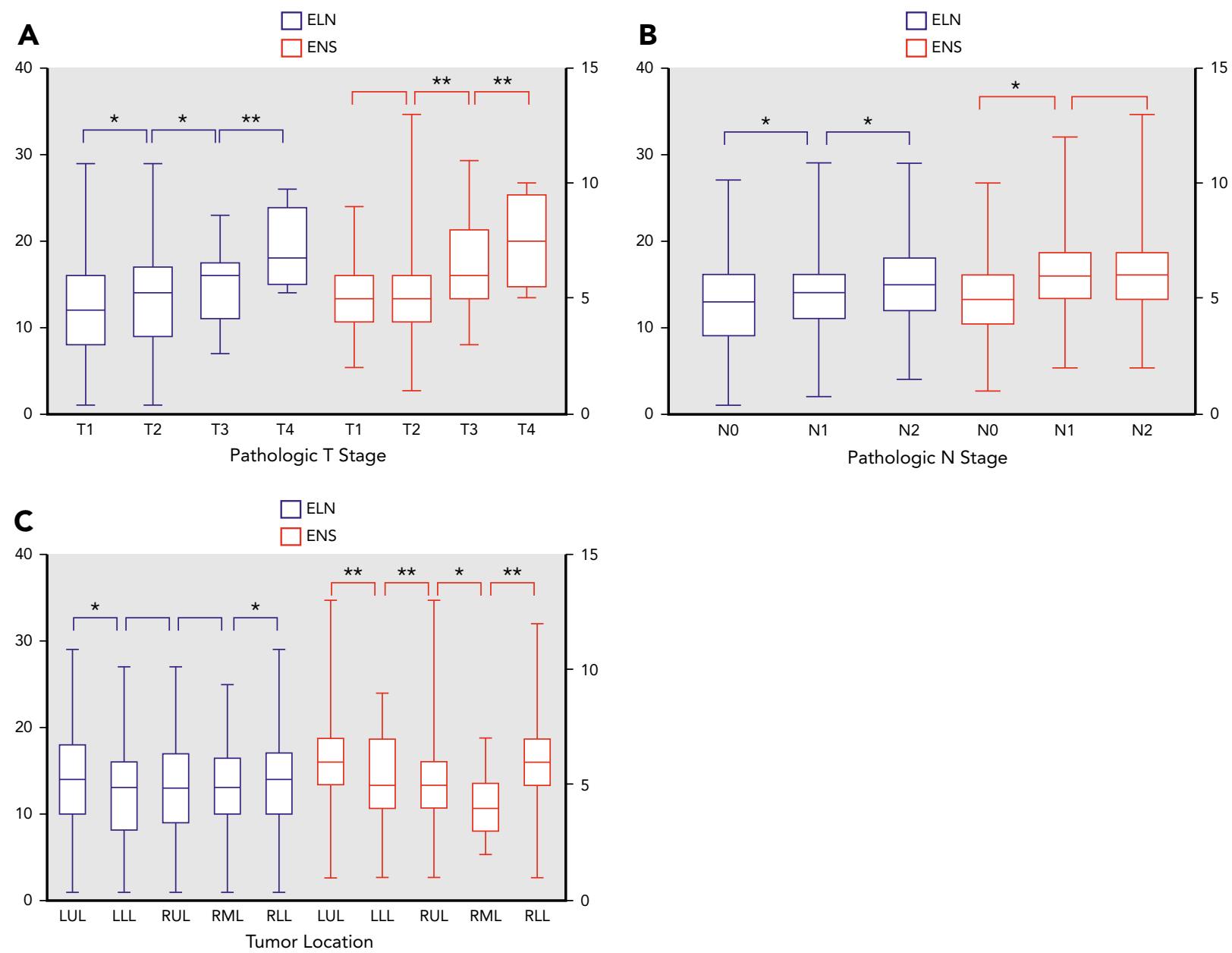

eFigure 3. Number of ELNs and ENSs among different (A) T stages, (B) N stages, and (C) and tumor locations.

Abbreviations: ELN, examined lymph node; ENS, examined node station; LLL, left lower lobe; LUL, left upper lobe; NSCLC, non-small cell lung cancer; RLL, right lower lobe; RML, right middle lobe; RUL, right upper lobe.

$\star P<.05$.

$\star \star P<.01$. 
A

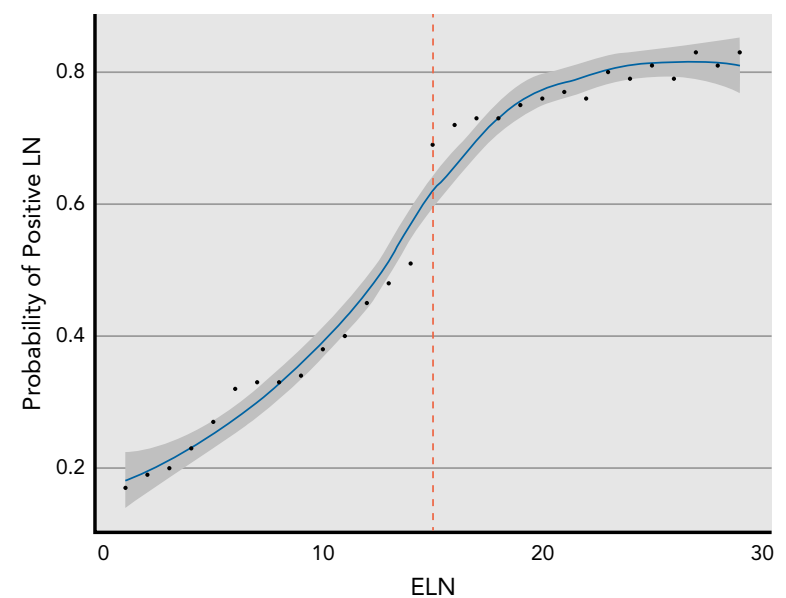

B

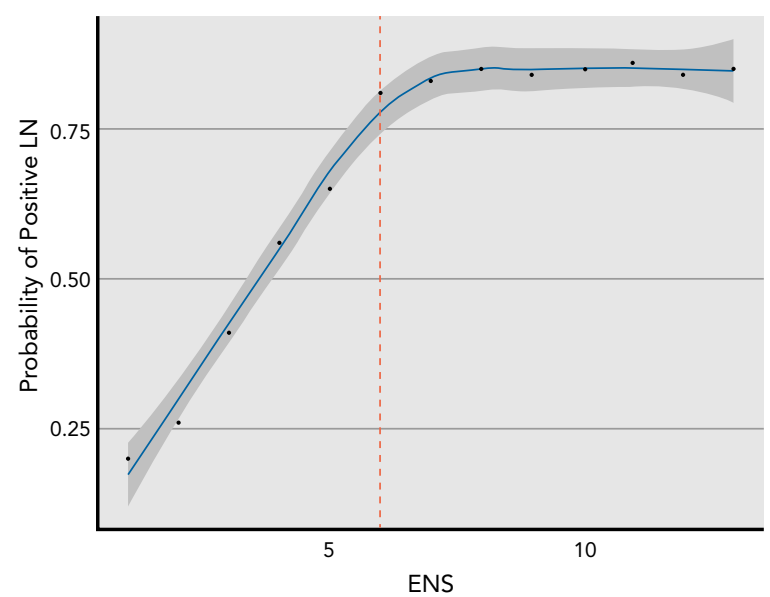

eFigure 4. Locally weighted least squares smoothing of percentage of patients with at least one positive LN or one involved node station as a function of the number of (A) ELNs and (B) ENSs, respectively.

Abbreviations: ELN, examined lymph node; ENS, examined node station; LN, lymph node.

A

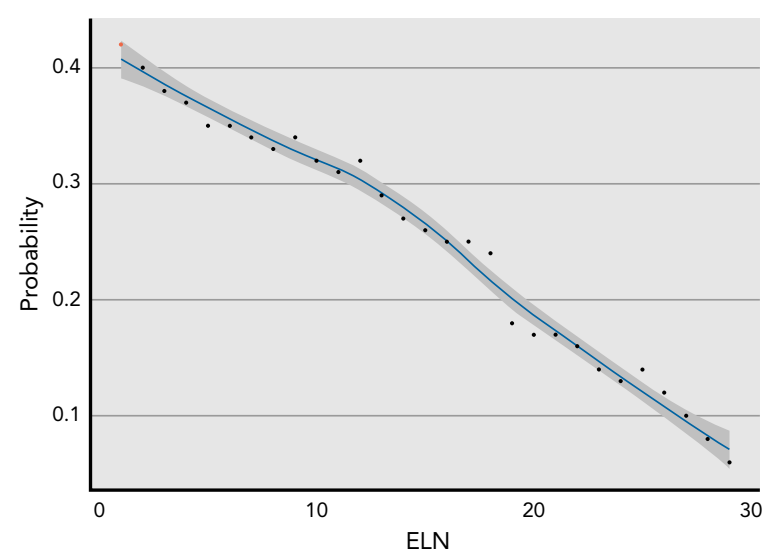

C

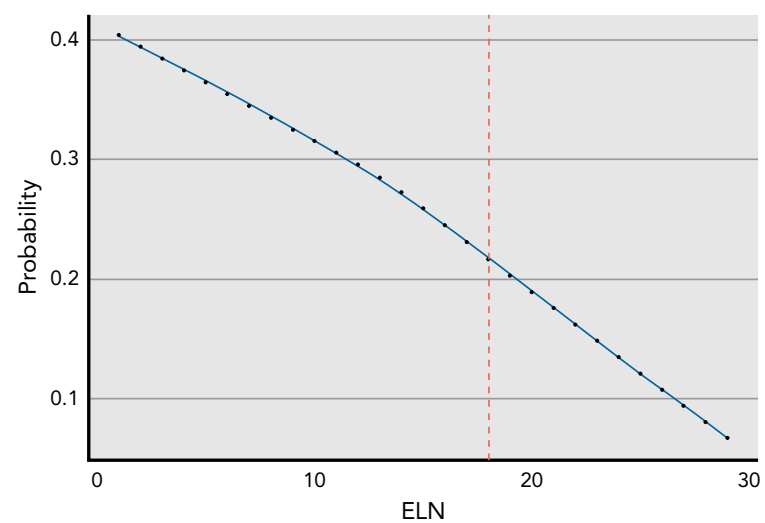

B

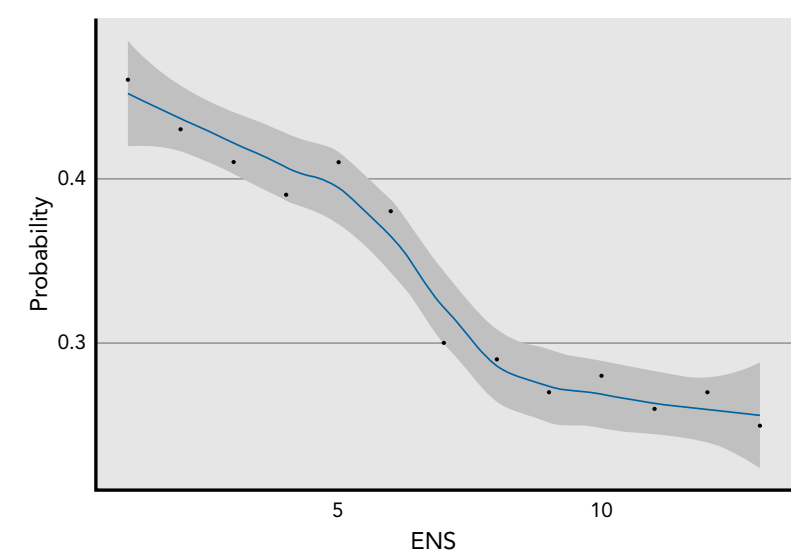

D

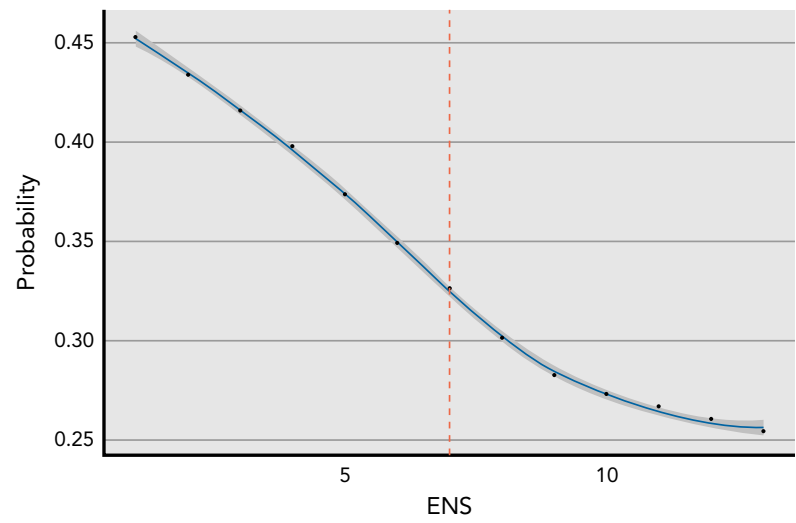

eFigure 5. LOWESS smoother fitting curves of estimated probabilities of (A, B) undetected positive ELNs or ENSs and (C, D) determination of structural break points using the Chow test in patients with declared node-negative (pNO) disease.

Abbreviations: ELN, examined lymph node; ENS, examined node station; LOWESS, locally weighted scatterplot smoothing. 
A

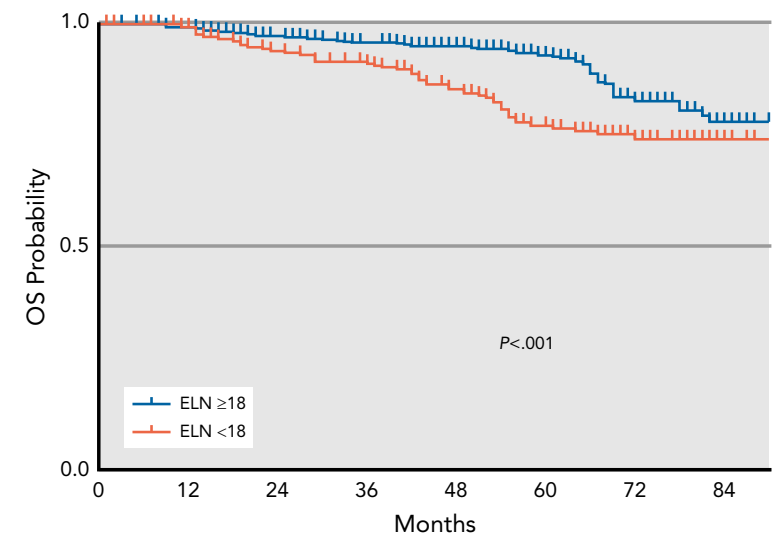

Number at risk

ELN $<18 \quad 972$

$\mathrm{ELN} \geq 18 \quad 233$
C

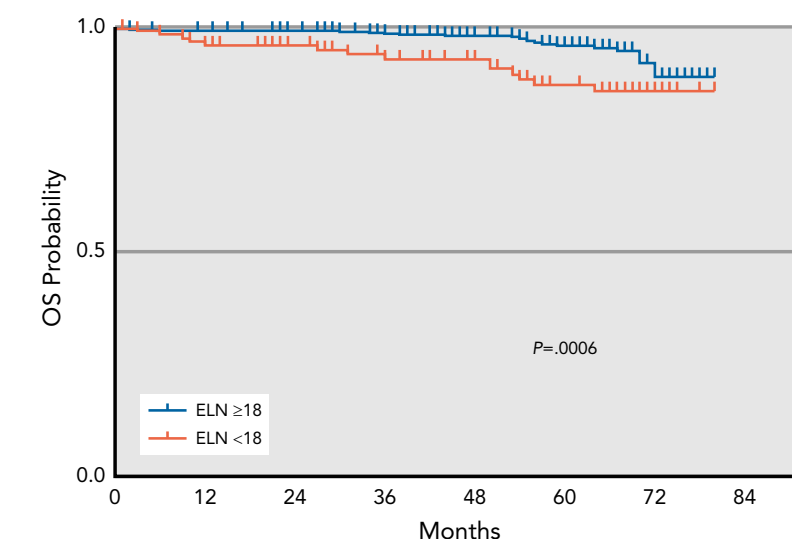

Number at risk

ELN $<18 \quad 757$

$\mathrm{ELN} \geq 18 \quad 151$

$$
75
$$

B

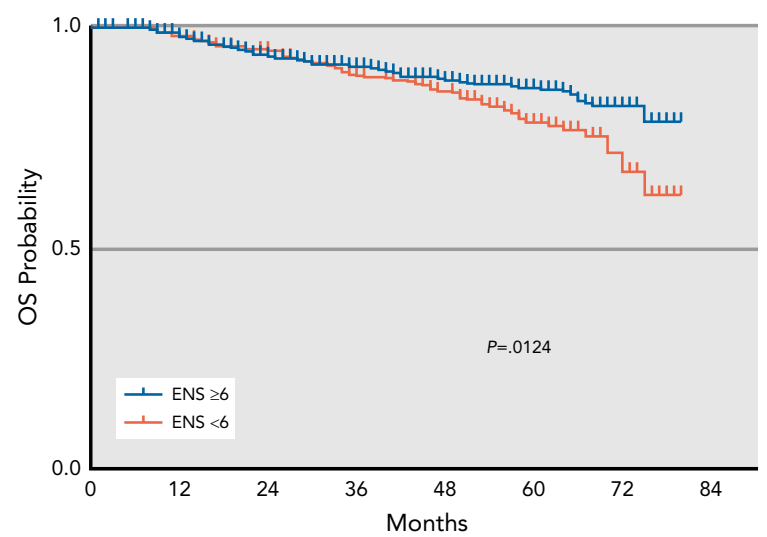

$\begin{array}{lrlllllll}\text { Number at risk } & & & & & & \\ \text { ENS }<6 & 665 & 657 & 631 & 618 & 601 & 577 & 576 & 573 \\ \text { ENS } \geq 6 & 540 & 525 & 514 & 496 & 485 & 480 & 477 & 472\end{array}$

D

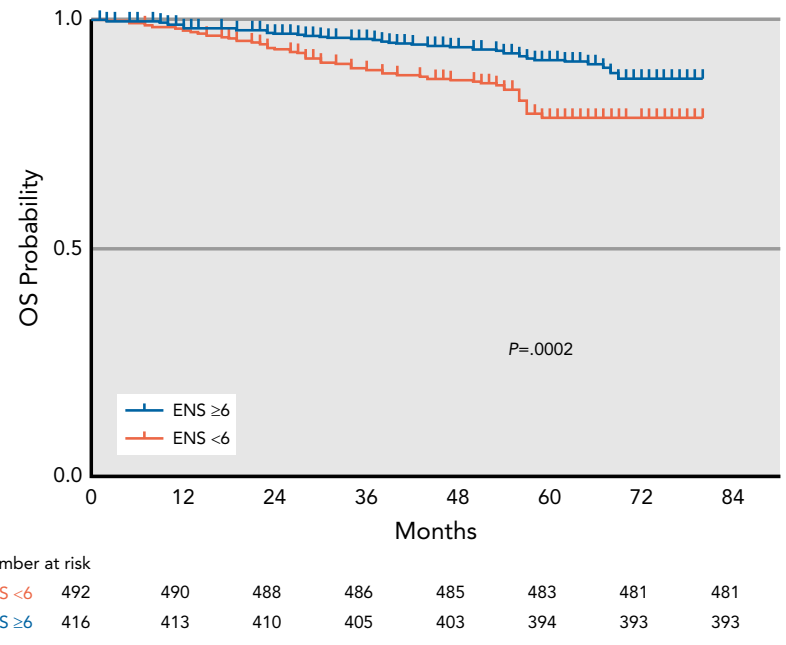

eFigure 6. Stratification of OS at the cut point of the number of ELNs and ENSs in $(A, B)$ the entire patient cohort and (C, D) patients with declared node-negative ( $\mathrm{pN} 0$ ) disease.

Abbreviations: ELN, examined lymph node; ENS, examined node station; OS, overall survival. 


\section{eTable 1. Baseline Characteristics of the Patient} Cohort $(\mathrm{N}=1,205)$

\begin{tabular}{|c|c|}
\hline Characteristic & n (\%) \\
\hline \multicolumn{2}{|l|}{ Sex } \\
\hline Male & 701 (58.17) \\
\hline Female & $504(41.83)$ \\
\hline \multicolumn{2}{|l|}{ Age, y } \\
\hline$\leq 60$ & $626(51.95)$ \\
\hline$>60$ & $579(48.05)$ \\
\hline \multicolumn{2}{|l|}{ Smoking history } \\
\hline Yes & 472 (39.17) \\
\hline No & $733(60.83)$ \\
\hline \multicolumn{2}{|l|}{ Operated side } \\
\hline Left & $557(46.22)$ \\
\hline Right & $648(53.78)$ \\
\hline \multicolumn{2}{|l|}{ Tumor location } \\
\hline Upper lobe & 731 (60.66) \\
\hline Middle lobe & $66(5.48)$ \\
\hline Lower lobe & $408(33.86)$ \\
\hline \multicolumn{2}{|l|}{ EBUS-TBNA } \\
\hline Yes & $726(60.25)$ \\
\hline No & 479 (39.75) \\
\hline \multicolumn{2}{|l|}{ Mediastinoscopy } \\
\hline Yes & $131(10.87)$ \\
\hline No & $1,074(89.13)$ \\
\hline \multicolumn{2}{|l|}{ cT } \\
\hline $\mathrm{T} 1$ & $438(36.35)$ \\
\hline $\mathrm{T} 2$ & 767 (63.65) \\
\hline \multicolumn{2}{|l|}{$\mathrm{cN}$} \\
\hline No & 1,078 (89.46) \\
\hline N1 & $127(10.54)$ \\
\hline \multicolumn{2}{|l|}{ pT } \\
\hline $\mathrm{T} 1$ & 577 (47.88) \\
\hline $\mathrm{T} 2$ & $614(50.95)$ \\
\hline T3 & $10(0.83)$ \\
\hline $\mathrm{T} 4$ & $4(0.33)$ \\
\hline \multicolumn{2}{|l|}{$\mathrm{pN}$} \\
\hline No & 908 (75.35) \\
\hline N1 & $131(10.87)$ \\
\hline N2 & 166 (13.78) \\
\hline
\end{tabular}

(continued)

\section{eTable 1. Baseline Characteristics of the Patient} Cohort $(N=1,205)$ (cont.)

\section{Characteristic}

n (\%)

PTNM

la $\quad 460$ (38.17)

$\mathrm{lb}$ $408(33.86)$

Ila

$74(6.14)$

$\mathrm{llb}$

87 (7.22)

IIla

$173(14.36)$

IIIb

$1(0.08)$

IV

$2(0.17)$

Histologic type

\begin{tabular}{lc} 
Squamous cell carcinoma & $226(18.76)$ \\
\hline Adenocarcinoma & $884(73.36)$ \\
\hline Adenosquamous carcinoma & $41(3.40)$ \\
\hline Large cell carcinoma & $31(2.57)$ \\
\hline Others & $23(1.91)$
\end{tabular}

Grade of differentiation

\begin{tabular}{ll} 
Well differentiated & $80(6.64)$ \\
\hline Moderately differentiated & $723(60)$ \\
\hline Poorly differentiated & $233(19.34)$ \\
\hline Not determined & $169(14.02)$
\end{tabular}

Visceral pleural involvement

\begin{tabular}{ll}
\hline Yes & $450(37.34)$ \\
\hline No & 755 (62.66)
\end{tabular}

Surgical approach

\begin{tabular}{lr}
\hline VATS & $1,068(88.63)$ \\
\hline Thoracotomy & $137(11.37)$
\end{tabular}

Number of $\mathrm{N} 1$ nodes examined

$\begin{array}{ll}\leq 4 & 632(52.45) \\ >4 & 573(47.55)\end{array}$

Positive N1 nodes

\begin{tabular}{ll}
\hline Yes & $228(18.92)$ \\
\hline No & $977(81.08)$
\end{tabular}

Number of N2 nodes examined

\begin{tabular}{ll}
$\leq 7$ & $605(50.21)$ \\
\hline$>7$ & $600(49.79)$
\end{tabular}

Positive N2 nodes

\begin{tabular}{lr}
\hline Yes & $169(14.02)$ \\
\hline No & $1,036(85.98)$
\end{tabular}

$1,036(85.98)$

Total number of examined nodes

\begin{tabular}{ll}
$\leq 11$ & $493(40.91)$ \\
\hline$>11$ & $712(59.09)$
\end{tabular}

(continued on next page) 


\section{eTable 1. Baseline Characteristics of the Patient} Cohort $(\mathrm{N}=1,205)$ (cont.)

\section{Characteristic}

n (\%)

Positive lymph nodes

Yes 299 (24.81)

No 906 (75.19)

Adjuvant chemotherapy

\begin{tabular}{ll} 
Yes & $628(52.12)$ \\
\hline$N o$ & $577(47.88)$
\end{tabular}

No

$62(5.15)$

Adjuvant radiotherapy

\begin{tabular}{lc}
\hline Yes & $62(5.15)$ \\
\hline No & $1,143(94.85)$
\end{tabular}

Locoregional recurrence

\begin{tabular}{ll}
\hline Yes & $232(19.25)$ \\
\hline No & $973(80.75)$
\end{tabular}

Distant metastasis

Yes 184 (15.27)

No $1,021(84.73)$

$\mathrm{cN} / \mathrm{pN}$ upstaging

Yes 170 (14.11)

No 1,035 (85.89)

Abbreviations: c, clinical; EBUS-TBNA, endobronchial ultrasound-guided transbronchial needle aspiration; $p$, pathologic; VATS, video-assisted thoracoscopic surgery. 
eTable 2. Variants in LASSO Regression Analysis of Screening Data for Survival Analysis

\section{Characteristics}

Variant Attributes

\begin{tabular}{|ll|}
\hline Sex & Categorical \\
\hline Age & Continuous \\
\hline Smoking history & Categorical \\
\hline Operated side & Categorical \\
\hline Tumor location & Categorical \\
\hline pT & Categorical \\
\hline pN & Categorical \\
\hline pM & Categorical \\
\hline Histologic type & Categorical \\
\hline Grade of differentiation & Categorical \\
\hline Surgical approach & Categorical \\
\hline Number of N1 stations & Continuous \\
\hline Number of N1 nodes examined & Continuous \\
\hline Number of N2 stations & Continuous \\
\hline Number of N2 nodes examined & Continuous \\
\hline Adjuvant chemotherapy & Categorical \\
\hline Adjuvant radiotherapy & Categorical \\
\hline Total number of stations & Continuous \\
\hline Total number of examined nodes & Continuous \\
\hline Lymph node ratio & Continuous \\
\hline
\end{tabular}

Abbreviation: $p$, pathologic.

\section{eTable 3. Variants in LASSO Regression Analysis of Screening Data for Multivariate Logistic Regression Analysis}

\begin{tabular}{|ll|}
\hline Characteristics & Variant Attributes \\
\hline Sex & Categorical \\
\hline Age & Continuous \\
\hline Smoking history & Categorical \\
Operated side & Categorical \\
\hline Tumor location & Categorical \\
\hline pT & Categorical \\
\hline EBUS-TBNA & Categorical \\
\hline Mediastinoscopy & Categorical \\
\hline Histologic type & Categorical \\
\hline Grade of differentiation & Categorical \\
\hline Surgical approach & Categorical \\
\hline Number of N1 stations & Continuous \\
\hline Number of N1 nodes examined & Continuous \\
\hline Number of N2 stations & Continuous \\
\hline Number of N2 nodes examined & Continuous \\
\hline Total number of stations & Continuous \\
\hline Total number of examined nodes & Continuous \\
\hline
\end{tabular}

Abbreviations: EBUS-TBNA, endobronchial ultrasound-guided transbronchial needle aspiration; $p$, pathologic. 
eTable 4. Accuracy of Number of Positive Nodes

No. of Positive Nodes Observed

\begin{tabular}{|c|c|c|c|c|c|c|c|c|c|c|c|c|c|}
\hline \multirow{2}{*}{$\begin{array}{l}\text { No. of Harvested } \\
\text { Lymph Nodes }\end{array}$} & \\
\hline & 1 & 2 & 3 & 4 & 5 & 6 & 7 & 8 & 9 & 10 & 11 & 12 & 13 \\
\hline 1 & $12.42 \%$ & & & & & & & & & & & & \\
\hline 2 & $14.21 \%$ & $6.06 \%$ & & & & & & & & & & & \\
\hline 3 & $16.04 \%$ & $7.66 \%$ & $1.51 \%$ & & & & & & & & & & \\
\hline 4 & $17.90 \%$ & $9.50 \%$ & $2.08 \%$ & $0.64 \%$ & & & & & & & & & \\
\hline 5 & $19.79 \%$ & $11.52 \%$ & $2.83 \%$ & $0.90 \%$ & $0.26 \%$ & & & & & & & & \\
\hline 6 & $21.72 \%$ & $13.70 \%$ & $3.80 \%$ & $1.28 \%$ & $0.36 \%$ & $0.10 \%$ & & & & & & & \\
\hline 7 & $23.70 \%$ & $15.99 \%$ & $5.01 \%$ & $1.81 \%$ & $0.51 \%$ & $0.13 \%$ & $0.02 \%$ & & & & & & \\
\hline 8 & $25.74 \%$ & $18.36 \%$ & $6.46 \%$ & $2.57 \%$ & $0.72 \%$ & $0.18 \%$ & $0.03 \%$ & $0.01 \%$ & & & & & \\
\hline 9 & $27.86 \%$ & $20.82 \%$ & $8.14 \%$ & $3.61 \%$ & $1.04 \%$ & $0.26 \%$ & $0.03 \%$ & $0.01 \%$ & $<0.01 \%$ & & & & \\
\hline 10 & $30.06 \%$ & $23.36 \%$ & $10.01 \%$ & $5.02 \%$ & $1.50 \%$ & $0.36 \%$ & $0.05 \%$ & $0.03 \%$ & $0.01 \%$ & $<0.01 \%$ & & & \\
\hline 11 & $32.36 \%$ & $26.00 \%$ & $12.04 \%$ & $6.85 \%$ & $2.19 \%$ & $0.52 \%$ & $0.06 \%$ & $0.03 \%$ & $0.01 \%$ & $<0.01 \%$ & $<0.01 \%$ & & \\
\hline 12 & $34.77 \%$ & $28.76 \%$ & $14.20 \%$ & $9.15 \%$ & $3.19 \%$ & $0.75 \%$ & $0.09 \%$ & $0.05 \%$ & $0.03 \%$ & $0.01 \%$ & $<0.01 \%$ & $<0.01 \%$ & \\
\hline 13 & $37.29 \%$ & $31.65 \%$ & $16.47 \%$ & $11.90 \%$ & $4.64 \%$ & $1.10 \%$ & $0.12 \%$ & $0.09 \%$ & $0.05 \%$ & $0.03 \%$ & $0.01 \%$ & $<0.01 \%$ & $<0.01 \%$ \\
\hline 14 & $39.92 \%$ & $34.70 \%$ & $18.86 \%$ & $15.01 \%$ & $6.69 \%$ & $1.63 \%$ & $0.18 \%$ & $0.13 \%$ & $0.10 \%$ & $0.07 \%$ & $0.05 \%$ & $0.03 \%$ & $<0.01 \%$ \\
\hline 15 & $42.69 \%$ & $37.92 \%$ & $21.38 \%$ & $18.41 \%$ & $9.51 \%$ & $2.43 \%$ & $0.26 \%$ & $0.19 \%$ & $0.15 \%$ & $0.11 \%$ & $0.07 \%$ & $0.03 \%$ & $0.01 \%$ \\
\hline 16 & $45.58 \%$ & $41.32 \%$ & $24.08 \%$ & $22.00 \%$ & $13.19 \%$ & $3.66 \%$ & $0.38 \%$ & $0.25 \%$ & $0.19 \%$ & $0.15 \%$ & $0.11 \%$ & $0.08 \%$ & $0.03 \%$ \\
\hline 17 & $48.61 \%$ & $44.92 \%$ & $27.00 \%$ & $25.72 \%$ & $17.77 \%$ & $5.50 \%$ & $0.57 \%$ & $0.32 \%$ & $0.25 \%$ & $0.19 \%$ & $0.15 \%$ & $0.11 \%$ & $0.06 \%$ \\
\hline 18 & $51.79 \%$ & $48.72 \%$ & $30.18 \%$ & $29.57 \%$ & $23.12 \%$ & $8.24 \%$ & $0.87 \%$ & $0.54 \%$ & $0.32 \%$ & $0.24 \%$ & $0.19 \%$ & $0.16 \%$ & $0.10 \%$ \\
\hline 19 & $55.12 \%$ & $52.71 \%$ & $33.67 \%$ & $33.56 \%$ & $29.05 \%$ & $12.17 \%$ & $1.34 \%$ & $0.88 \%$ & $0.59 \%$ & $0.36 \%$ & $0.25 \%$ & $0.21 \%$ & $0.16 \%$ \\
\hline 20 & $58.61 \%$ & $56.89 \%$ & $37.54 \%$ & $37.75 \%$ & $35.30 \%$ & $17.61 \%$ & $2.07 \%$ & $1.41 \%$ & $0.91 \%$ & $0.62 \%$ & $0.38 \%$ & $0.26 \%$ & $0.20 \%$ \\
\hline 21 & $62.27 \%$ & $61.24 \%$ & $41.83 \%$ & $42.22 \%$ & $41.69 \%$ & $24.76 \%$ & $3.20 \%$ & $2.12 \%$ & $1.52 \%$ & $0.96 \%$ & $0.64 \%$ & $0.41 \%$ & $0.29 \%$ \\
\hline 22 & $66.12 \%$ & $65.77 \%$ & $46.61 \%$ & $47.05 \%$ & $48.09 \%$ & $33.64 \%$ & $4.93 \%$ & $3.27 \%$ & $2.35 \%$ & $1.61 \%$ & $1.16 \%$ & $0.74 \%$ & $0.43 \%$ \\
\hline 23 & $70.17 \%$ & $70.43 \%$ & $51.95 \%$ & $52.33 \%$ & $54.49 \%$ & $43.99 \%$ & $7.50 \%$ & $5.12 \%$ & $3.74 \%$ & $2.69 \%$ & $1.98 \%$ & $1.28 \%$ & $0.89 \%$ \\
\hline 24 & $74.44 \%$ & $75.22 \%$ & $57.91 \%$ & $58.17 \%$ & $60.93 \%$ & $55.32 \%$ & $11.26 \%$ & $7.92 \%$ & $5.87 \%$ & $4.02 \%$ & $2.81 \%$ & $2.02 \%$ & $1.35 \%$ \\
\hline 25 & $78.95 \%$ & $80.10 \%$ & $64.59 \%$ & $64.69 \%$ & $67.54 \%$ & $66.95 \%$ & $16.72 \%$ & $12.56 \%$ & $8.15 \%$ & $6.16 \%$ & $4.27 \%$ & $2.98 \%$ & $2.41 \%$ \\
\hline 26 & $83.72 \%$ & $85.05 \%$ & $72.04 \%$ & $72.00 \%$ & $74.46 \%$ & $78.02 \%$ & $24.76 \%$ & $18.96 \%$ & $14.87 \%$ & $9.52 \%$ & $7.81 \%$ & $4.52 \%$ & $3.82 \%$ \\
\hline 27 & $88.80 \%$ & $90.03 \%$ & $80.37 \%$ & $80.24 \%$ & $81.94 \%$ & $87.63 \%$ & $37.34 \%$ & $26.12 \%$ & $19.12 \%$ & $15.72 \%$ & $10.63 \%$ & $8.75 \%$ & $6.03 \%$ \\
\hline 28 & $94.21 \%$ & $95.03 \%$ & $89.66 \%$ & $89.54 \%$ & $90.30 \%$ & $95.01 \%$ & $59.14 \%$ & $39.34 \%$ & $28.25 \%$ & $21.62 \%$ & $16.77 \%$ & $12.06 \%$ & $8.89 \%$ \\
\hline 29 & $100.00 \%$ & $100.00 \%$ & $100.00 \%$ & $100.00 \%$ & $100.00 \%$ & $100.00 \%$ & $100.00 \%$ & $65.27 \%$ & $41.28 \%$ & $30.58 \%$ & $22.81 \%$ & $16.89 \%$ & $10.02 \%$ \\
\hline
\end{tabular}




\section{eTable 5. Accuracy of Number of Positive Stations}

No. of Positive Nodes Observed

\begin{tabular}{|c|c|c|c|c|c|c|}
\hline \multirow[b]{2}{*}{ No. of Node Stations Examined } & & & & & & \\
\hline & 1 & 2 & 3 & 4 & 5 & 6 \\
\hline 1 & $32.10 \%$ & & & & & \\
\hline 2 & $37.01 \%$ & $24.17 \%$ & & & & \\
\hline 3 & $47.10 \%$ & $28.26 \%$ & $16.11 \%$ & & & \\
\hline 4 & $48.08 \%$ & $33.00 \%$ & $19.03 \%$ & $18.25 \%$ & & \\
\hline 5 & $49.84 \%$ & $38.39 \%$ & $22.64 \%$ & $20.75 \%$ & $17.33 \%$ & \\
\hline 6 & $54.98 \%$ & $44.47 \%$ & $27.13 \%$ & $23.83 \%$ & $19.87 \%$ & $15.54 \%$ \\
\hline 7 & $60.39 \%$ & $51.19 \%$ & $32.71 \%$ & $27.65 \%$ & $22.53 \%$ & $20.37 \%$ \\
\hline 8 & $66.07 \%$ & $58.47 \%$ & $39.66 \%$ & $32.69 \%$ & $27.71 \%$ & $26.65 \%$ \\
\hline 9 & $72.06 \%$ & $66.22 \%$ & $48.25 \%$ & $38.76 \%$ & $33.54 \%$ & $31.12 \%$ \\
\hline 10 & $78.40 \%$ & $74.31 \%$ & $58.71 \%$ & $47.10 \%$ & $40.43 \%$ & $38.87 \%$ \\
\hline 11 & $85.13 \%$ & $82.65 \%$ & $71.12 \%$ & $58.56 \%$ & $51.31 \%$ & $46.25 \%$ \\
\hline 12 & $92.31 \%$ & $91.19 \%$ & $85.18 \%$ & $75.00 \%$ & $69.02 \%$ & $63.87 \%$ \\
\hline 13 & $100.00 \%$ & $100.00 \%$ & $100.00 \%$ & $100.00 \%$ & $100.00 \%$ & $100.00 \%$ \\
\hline
\end{tabular}


eTable 6. Probability of Having M+1 or More Positive Nodes Given the Observation of M-Positive Nodes in $\mathbf{N}$-Resected Nodes

\begin{tabular}{|c|c|c|c|c|c|c|c|c|c|c|c|c|c|c|}
\hline \multirow{2}{*}{$\begin{array}{l}\text { No. of } \\
\text { Harvested } \\
\text { Lymph } \\
\text { Nodes (N) }\end{array}$} & \multicolumn{14}{|c|}{ No. of Positive Nodes Observed } \\
\hline & 0 & 1 & 2 & 3 & 4 & 5 & 6 & 7 & 8 & 9 & 10 & 11 & 12 & 13 \\
\hline 1 & $40.12 \%$ & $47.51 \%$ & & & & & & & & & & & & \\
\hline 2 & $38.59 \%$ & $43.03 \%$ & $87.76 \%$ & & & & & & & & & & & \\
\hline 3 & $37.26 \%$ & $39.87 \%$ & $83.64 \%$ & $99.23 \%$ & & & & & & & & & & \\
\hline 4 & $36.08 \%$ & $36.32 \%$ & $78.08 \%$ & $97.92 \%$ & $100.00 \%$ & & & & & & & & & \\
\hline 5 & $34.87 \%$ & $33.44 \%$ & $74.01 \%$ & $97.17 \%$ & $100.00 \%$ & $100.00 \%$ & & & & & & & & \\
\hline 6 & $33.25 \%$ & $29.58 \%$ & $69.82 \%$ & $96.20 \%$ & $100.00 \%$ & $100.00 \%$ & $100.00 \%$ & & & & & & & \\
\hline 7 & $32.17 \%$ & $25.09 \%$ & $65.61 \%$ & $94.99 \%$ & $100.00 \%$ & $100.00 \%$ & $100.00 \%$ & $100.00 \%$ & & & & & & \\
\hline 8 & $31.09 \%$ & $22.17 \%$ & $61.46 \%$ & $93.54 \%$ & $100.00 \%$ & $100.00 \%$ & $100.00 \%$ & $100.00 \%$ & $100.00 \%$ & & & & & \\
\hline 9 & $29.57 \%$ & $19.92 \%$ & $57.39 \%$ & $91.86 \%$ & $100.00 \%$ & $100.00 \%$ & $100.00 \%$ & $100.00 \%$ & $100.00 \%$ & $100.00 \%$ & & & & \\
\hline 10 & $28.63 \%$ & $17.50 \%$ & $53.41 \%$ & $89.99 \%$ & $100.00 \%$ & $100.00 \%$ & $100.00 \%$ & $100.00 \%$ & $100.00 \%$ & $100.00 \%$ & $100.00 \%$ & & & \\
\hline 11 & $27.54 \%$ & $15.54 \%$ & $49.50 \%$ & $87.96 \%$ & $100.00 \%$ & $100.00 \%$ & $100.00 \%$ & $100.00 \%$ & $100.00 \%$ & $100.00 \%$ & $100.00 \%$ & $100.00 \%$ & & \\
\hline 12 & $26.07 \%$ & $13.47 \%$ & $44.95 \%$ & $85.80 \%$ & $100.00 \%$ & $100.00 \%$ & $100.00 \%$ & $100.00 \%$ & $100.00 \%$ & $100.00 \%$ & $100.00 \%$ & $100.00 \%$ & $100.00 \%$ & \\
\hline 13 & $24.84 \%$ & $11.52 \%$ & $41.48 \%$ & $83.53 \%$ & $100.00 \%$ & $100.00 \%$ & $100.00 \%$ & $100.00 \%$ & $100.00 \%$ & $100.00 \%$ & $100.00 \%$ & $100.00 \%$ & $100.00 \%$ & $100.00 \%$ \\
\hline 14 & $23.18 \%$ & $9.71 \%$ & $37.14 \%$ & $79.53 \%$ & $100.00 \%$ & $100.00 \%$ & $100.00 \%$ & $100.00 \%$ & $100.00 \%$ & $100.00 \%$ & $100.00 \%$ & $100.00 \%$ & $100.00 \%$ & $100.00 \%$ \\
\hline 15 & $21.76 \%$ & $8.02 \%$ & $33.45 \%$ & $77.38 \%$ & $100.00 \%$ & $100.00 \%$ & $100.00 \%$ & $100.00 \%$ & $100.00 \%$ & $100.00 \%$ & $100.00 \%$ & $100.00 \%$ & $100.00 \%$ & $100.00 \%$ \\
\hline 16 & $20.03 \%$ & $6.52 \%$ & $30.25 \%$ & $75.30 \%$ & $100.00 \%$ & $100.00 \%$ & $100.00 \%$ & $100.00 \%$ & $100.00 \%$ & $100.00 \%$ & $100.00 \%$ & $100.00 \%$ & $100.00 \%$ & $100.00 \%$ \\
\hline 17 & $18.72 \%$ & $5.19 \%$ & $26.70 \%$ & $72.16 \%$ & $97.54 \%$ & $100.00 \%$ & $100.00 \%$ & $100.00 \%$ & $100.00 \%$ & $100.00 \%$ & $100.00 \%$ & $100.00 \%$ & $100.00 \%$ & $100.00 \%$ \\
\hline 18 & $17.64 \%$ & $4.02 \%$ & $23.16 \%$ & $68.66 \%$ & $92.13 \%$ & $100.00 \%$ & $100.00 \%$ & $100.00 \%$ & $100.00 \%$ & $100.00 \%$ & $100.00 \%$ & $100.00 \%$ & $100.00 \%$ & $100.00 \%$ \\
\hline 19 & $16.32 \%$ & $3.02 \%$ & $19.62 \%$ & $65.68 \%$ & $86.71 \%$ & $100.00 \%$ & $100.00 \%$ & $100.00 \%$ & $100.00 \%$ & $100.00 \%$ & $100.00 \%$ & $100.00 \%$ & $100.00 \%$ & $100.00 \%$ \\
\hline 20 & $15.11 \%$ & $2.18 \%$ & $16.28 \%$ & $62.13 \%$ & $78.12 \%$ & $96.57 \%$ & $100.00 \%$ & $100.00 \%$ & $100.00 \%$ & $100.00 \%$ & $100.00 \%$ & $100.00 \%$ & $100.00 \%$ & $100.00 \%$ \\
\hline 21 & $13.69 \%$ & $1.48 \%$ & $13.10 \%$ & $58.02 \%$ & $70.39 \%$ & $92.36 \%$ & $100.00 \%$ & $100.00 \%$ & $100.00 \%$ & $100.00 \%$ & $100.00 \%$ & $100.00 \%$ & $100.00 \%$ & $100.00 \%$ \\
\hline 22 & $12.25 \%$ & $0.96 \%$ & $10.14 \%$ & $53.33 \%$ & $63.44 \%$ & $88.57 \%$ & $100.00 \%$ & $100.00 \%$ & $100.00 \%$ & $100.00 \%$ & $100.00 \%$ & $100.00 \%$ & $100.00 \%$ & $100.00 \%$ \\
\hline 23 & $11.03 \%$ & $0.57 \%$ & $7.45 \%$ & $48.04 \%$ & $58.56 \%$ & $81.65 \%$ & $100.00 \%$ & $100.00 \%$ & $100.00 \%$ & $100.00 \%$ & $100.00 \%$ & $100.00 \%$ & $100.00 \%$ & $100.00 \%$ \\
\hline 24 & $9.73 \%$ & $0.29 \%$ & $5.10 \%$ & $42.08 \%$ & $52.19 \%$ & $76.58 \%$ & $93.59 \%$ & $100.00 \%$ & $100.00 \%$ & $100.00 \%$ & $100.00 \%$ & $100.00 \%$ & $100.00 \%$ & $100.00 \%$ \\
\hline 25 & $8.11 \%$ & $0.12 \%$ & $3.13 \%$ & $35.41 \%$ & $47.54 \%$ & $71.26 \%$ & $89.01 \%$ & $100.00 \%$ & $100.00 \%$ & $100.00 \%$ & $100.00 \%$ & $100.00 \%$ & $100.00 \%$ & $100.00 \%$ \\
\hline 26 & $7.23 \%$ & $0.03 \%$ & $1.60 \%$ & $27.96 \%$ & $39.47 \%$ & $67.87 \%$ & $83.16 \%$ & $100.00 \%$ & $100.00 \%$ & $100.00 \%$ & $100.00 \%$ & $100.00 \%$ & $100.00 \%$ & $100.00 \%$ \\
\hline 27 & $6.16 \%$ & $0.00 \%$ & $0.54 \%$ & $19.63 \%$ & $31.58 \%$ & $61.77 \%$ & $75.28 \%$ & $98.74 \%$ & $100.00 \%$ & $100.00 \%$ & $100.00 \%$ & $100.00 \%$ & $100.00 \%$ & $100.00 \%$ \\
\hline 28 & $5.23 \%$ & $0.00 \%$ & $0.00 \%$ & $10.35 \%$ & $24.36 \%$ & $56.21 \%$ & $70.68 \%$ & $96.12 \%$ & $100.00 \%$ & $100.00 \%$ & $100.00 \%$ & $100.00 \%$ & $100.00 \%$ & $100.00 \%$ \\
\hline 29 & $3.66 \%$ & $0.00 \%$ & $0.00 \%$ & $0.00 \%$ & $12.59 \%$ & $50.27 \%$ & $65.13 \%$ & $93.87 \%$ & $100.00 \%$ & $100.00 \%$ & $100.00 \%$ & $100.00 \%$ & $100.00 \%$ & $100.00 \%$ \\
\hline
\end{tabular}


eTable 7. Probability of Having M+1 or More Positive Node Stations Given the Observation of M Stations With Positive Nodes in S-Examined Node Stations

\begin{tabular}{|c|c|c|c|c|c|c|c|}
\hline No. of Node Stations Examined (S) & \multicolumn{7}{|c|}{ No. of Positive Nodes Observed } \\
\hline 1 & $44.55 \%$ & $49.55 \%$ & & & & & \\
\hline 2 & $43.21 \%$ & $47.52 \%$ & $55.72 \%$ & & & & \\
\hline 3 & $42.02 \%$ & $43.52 \%$ & $52.42 \%$ & $87.42 \%$ & & & \\
\hline 4 & $40.85 \%$ & $39.42 \%$ & $47.89 \%$ & $80.42 \%$ & $100.00 \%$ & & \\
\hline 6 & $37.69 \%$ & $33.15 \%$ & $39.75 \%$ & $63.28 \%$ & $100.00 \%$ & $100.00 \%$ & $100.00 \%$ \\
\hline 7 & $36.14 \%$ & $29.24 \%$ & $35.47 \%$ & $55.28 \%$ & $95.01 \%$ & $100.00 \%$ & $100.00 \%$ \\
\hline 8 & $34.95 \%$ & $26.72 \%$ & $29.54 \%$ & $41.32 \%$ & $87.21 \%$ & $92.10 \%$ & $100.00 \%$ \\
\hline 9 & $33.54 \%$ & $22.78 \%$ & $23.45 \%$ & $30.27 \%$ & $79.14 \%$ & $83.10 \%$ & $100.00 \%$ \\
\hline 10 & $32.01 \%$ & $19.12 \%$ & $20.01 \%$ & $14.22 \%$ & $56.78 \%$ & $67.20 \%$ & $79.70 \%$ \\
\hline
\end{tabular}

